\title{
CONVOLVED SUBSAMPLING ESTIMATION WITH APPLICATIONS TO BLOCK BOOTSTRAP
}

\author{
By Johannes Tewes ${ }^{1}$, Dimitris N. Politis And Daniel J. Nordman ${ }^{2}$ \\ Ruhr-Universität Bochum, University of California, San Diego and \\ Iowa State University
}

The block bootstrap approximates sampling distributions from dependent data by resampling data blocks. A fundamental problem is establishing its consistency for the distribution of a sample mean, as a prototypical statistic. We use a structural relationship with subsampling to characterize the bootstrap in a new and general manner. While subsampling and block bootstrap differ, the block bootstrap distribution of a sample mean equals that of a $k$-fold self-convolution of a subsampling distribution. Motivated by this, we provide simple necessary and sufficient conditions for a convolved subsampling estimator to produce a normal limit that matches the target of bootstrap estimation. These conditions may be linked to consistency properties of an original subsampling distribution, which are often obtainable under minimal assumptions. Through several examples, the results are shown to validate the block bootstrap for means under significantly weakened assumptions in many existing (and some new) dependence settings, which also addresses a standing conjecture of Politis, Romano and Wolf [Subsampling (1999) Springer]. Beyond sample means, convolved subsampling may not match the block bootstrap, but instead provides an alternative resampling estimator that may be of interest. Under minimal dependence conditions, results also broadly establish convolved subsampling for general statistics having normal limits.

1. Introduction. Subsampling and block bootstrap are two common nonparametric tools for statistical inference under dependence; see Politis, Romano and Wolf [29] and Lahiri [19], respectively, for monographs on these. Both aim to approximate distributions of statistics with correlated data, and both are data resampling methods that use blocks of neighboring observations to capture dependence. The subsampling approach of Politis and Romano [28] treats data blocks as small scale renditions of the original data, which provides replication of a statistic for estimating a sampling distribution. The block bootstrap differs philosophically by using data blocks as building material to recreate the original data. Essentially, data blocks are randomly selected and pasted together to reproduce a full-scale set of bootstrap data, as proposed by Künsch [17] and Liu and Singh [25] for extending

Received April 2017; revised February 2018.

${ }^{1}$ Supported by the Collaborative Research Center "Statistical modeling of nonlinear dynamic processes" (SFB 823, Teilprojekt C2) of the German Research Foundation (DFG).

${ }^{2}$ Supported in part by NSF DMS-1406747.

MSC2010 subject classifications. Primary 62G09; secondary 62G20, 62J05, 62M10.

Key words and phrases. Convolution, mixing, moving blocks, nonstationary. 
Efron's [11] bootstrap to time series. As noted in Politis, Romano and Wolf [29] (cf. Section 3.9), subsampling is often valid under weak assumptions about the dependent process, basically requiring that a nondegenerate (possibly nonnormal) limit exist for the sampling distribution being approximated. In contrast, the block bootstrap applies to mean-like statistics with normal limits and typically requires comparatively much stronger assumptions for its validity. Case-by-case treatments are commonly needed to validate the bootstrap across differing dependence conditions. However, while perhaps not widely recognized, subsampling can in fact be used to verify the block bootstrap in some cases, which is a theme of this work.

We investigate estimators defined by the $k$-fold self-convolution of a subsampling distribution, and establish a new and general theory for their consistency to normal limits. There are two basic motivations for considering such convolved subsampling. The first is that, in the fundamental case of sample means, the block bootstrap estimator is a $k$-fold self-convolution of a subsampling distribution (centered and normalized), where the level $k$ of convolution corresponds to the number of resampled blocks. This observation was originally noted by Politis, Romano and Wolf [29], who suggested this aspect as a potential technique for showing the validity of the bootstrap. Specifically, they conjectured that convolved subsampling might provide a route for establishing the block bootstrap under minimal conditions for nonstationary, strongly mixing processes, in analogy to bootstrap results existing for stationary, mixing series due to Radulović [30, 31]. For the bootstrap under dependence, the findings for the sample mean in $[30,31]$ have stood out as an exception, verifying the method under the same weak assumptions as subsampling (i.e., conditions essentially needed for a normal limit to exist). By investigating the convolved subsampling approach here, we can answer the above conjecture affirmatively. Moreover, we show convolved subsampling leads to a simple and unified procedure for establishing the block bootstrap for sample means under further types of processes and much weaker conditions than previously considered, such as linear time processes, long-memory sequences, (nonstationary) almost periodic time series and spatial fields. Hence, convolved subsampling estimation allows for bootstrap consistency under dependence to be generally extended under the same weak assumptions used by subsampling, containing the conclusions of Radulović $[30,31]$ for stationary time series as a special case.

While connections to the bootstrap are useful, our study of convolved subsampling estimation is intended to be broad, applying also to general statistics with normal limits and with arbitrary levels of convolution. Consistency results often do not require particular assumptions about the underlying dependent process, but are rather formulated in terms of mild convergence properties of the original subsampling distribution and its variance. Furthermore, we show that a consistent subsampling variance is not only sufficient, but essentially necessary, for the consistency of convolved subsampling (and the block bootstrap in some cases). Due to its importance, we also provide tools for verifying the consistency of subsampling variance estimators. 
For general statistics beyond the sample mean, the convolved subsampling distribution may differ from the block bootstrap, which relates to a second motivation for our development. That is, a general theory for convolved subsampling may be of interest in its own right, as the approach can be computationally less demanding than the block bootstrap while also potentially enhancing ordinary subsampling for approximating sampling distributions with normal limits. In fact, there has been recent interest in establishing generalized types of subsampling estimation for complicated statistics under various dependence structures, where numerical studies suggest such methods can exhibit better finite sample performance than standard subsampling when the target distribution is normal; for example, see Lenart [22] and Sharipov, Tewes and Wendler [32] for spectral estimates and U-statistics, respectively, with time series. While not formally recognized as such, however, these proposed methods are exactly convolved subsampling estimators. By exploiting this realization, our results can facilitate future work and allow such previous findings with generalized subsampling to be demonstrated in an alternative, simpler manner with weaker assumptions; see Section 5 for illustrations of the examples mentioned above.

Section 2 describes convolved subsampling estimation and its connection to block bootstrap. General distributional results for convolved subsampling are given in Section 3, while Section 4 presents some applications with differing dependence structures. Section 4.1 provides a broad result for convolved subsampling estimation with statistics from mixing time series. Under weak conditions, Sections 4.24.5 apply convolved subsampling for demonstrating the block bootstrap for sample means with nonstationary time series (Section 4.2 and the conjecture of Politis, Romano and Wolf [29]), linear time processes (Section 4.3), long-range dependence (Section 4.4) and spatial data (Section 4.5). Section 5 describes relationships to other recent work with generalized subsampling, and Section 6 provides a short treatment of independent data. A numerical study of subsampling, block bootstrap and convolved subsamplng appears in Section 7, while Section 8 contains concluding remarks. The proofs of main results are given in the Supplementary Material [15].

Finally, to be clear, we stress that a central advantage of classical subsampling is its validity for nonnormal limits (cf. Section 4.4), which convolved subsampling does not share. The convolution of a subsampling distribution essentially induces a sum of independently resampled terms so that, like the block bootstrap, reproducing a nonnormal limit is impossible. However, for approximating normal targets, convolved subsampling does inherit the applicability of subsampling under weak conditions with general statistics.

\section{Description of convolved subsampling estimators.}

2.1. Problem background and original subsampling estimation. Consider data $X_{1}, \ldots, X_{n}$ from a real-valued process equipped with a probability structure $P$. 
For concreteness, we may view such observations as arising from a time series process $\left\{X_{t}\right\}$, though spatial and other data schemes may be treated as well. Based on $X_{1}, \ldots, X_{n}$, consider the problem of approximating the distribution of

$$
T_{n} \equiv \tau_{n}\left(t_{n}\left(X_{1}, \ldots, X_{n}\right)-t(P)\right),
$$

involving an estimator $t_{n} \equiv t_{n}\left(X_{1}, \ldots, X_{n}\right)$ of a parameter $t(P)$ and a sequence of positive scaling factors $\tau_{n}$ yielding a distributional limit for $T_{n}$. For example, if $t_{n}\left(X_{1}, \ldots, X_{n}\right) \equiv \bar{X}_{n}=\sum_{i=1}^{n} X_{i} / n$ is the sample mean, then $t(P)$ may correspond to a common process mean $\mu$ and $T_{n}$ may be defined with usual scaling $\tau_{n}=\sqrt{n}$ under weak time dependence. Denote the sampling distribution function of $T_{n}$ as $F_{n}(x)=P\left(T_{n} \leq x\right), x \in \mathbb{R}$.

We next define the subsampling estimator of $F_{n}$; see [28]. For a positive integer $b \equiv b_{n}<n$, let $\left\{\left(X_{i}, \ldots, X_{i+b-1}\right): i=1, \ldots, N_{n}\right\}$ denote the set of $N_{n} \equiv n-b+1$ overlapping data blocks, or subsamples, of length $b$. To keep blocks relatively small, the block size is often assumed to satisfy $b^{-1}+b / n+\tau_{b} / \tau_{n} \rightarrow 0$ as $n \rightarrow$ $\infty$. For each subsample, we compute the statistic as $t_{n, b, i}=t_{b}\left(X_{i}, \ldots, X_{i-b+1}\right)$ and define a "scale $b$ " version of $T_{n} \equiv \tau_{n}\left(t_{n}\left(X_{1}, \ldots, X_{n}\right)-t(P)\right)$ as $\tau_{b}\left[t_{n, b, i}-\right.$ $t_{n}$ ] for $i=1, \ldots, N_{n}$. Letting $I(\cdot)$ denote the indicator function, the subsampling estimator of $F_{n}$ is given by

$$
S_{n, \mathrm{SUB}}(x)=\frac{1}{N_{n}} \sum_{i=1}^{N_{n}} I\left(\tau_{b}\left[t_{n, b, i}-t_{n}\right] \leq x\right), \quad x \in \mathbb{R},
$$

or the empirical distribution of subsample analogs $\left\{\tau_{b}\left[t_{n, b, i}-t_{n}\right]\right\}_{i=1}^{N_{n}}$ (cf. [29]).

Suppose that $S_{n, \mathrm{SUB}}$ is consistent for the distribution of $T_{n}$, which has an asymptotically normal $N\left(0, \sigma^{2}\right)$ limit for some $\sigma>0$, that is, as $n \rightarrow \infty$,

$$
\begin{aligned}
T_{n} & \stackrel{d}{\rightarrow} N\left(0, \sigma^{2}\right), \\
\sup _{x \in \mathbb{R}}\left|S_{n, \operatorname{SUB}}(x)-\Phi(x / \sigma)\right| & \stackrel{p}{\rightarrow} 0,
\end{aligned}
$$

where $\Phi(\cdot)$ is the standard normal distribution function. We wish to consider estimators of the distribution $F_{n}$ of $T_{n}$ formed by self-convolutions of the subsampling estimator $S_{n, \mathrm{SUB}}$. This provides a general class of block resampling estimators in its own right, but also has explicit connections to block bootstrap estimators in the important case that the statistic of interest $t_{n}\left(X_{1}, \ldots, X_{n}\right)=\bar{X}_{n}$ is a sample mean, as described next.

2.2. Convolved subsampling and connections to block bootstrap. Let $k_{n}$ be a sequence of positive integers and define a triangular array $\left\{Y_{n, 1}^{*}, \ldots, Y_{n, k_{n}}^{*}\right\}_{n \geq 1}$, where, for each $n,\left\{Y_{n, j}^{*}\right\}_{j=1}^{k_{n}}$ are i.i.d. variables following the subsampling distribution $S_{n, \mathrm{SUB}}$, as determined by (2.1) from data $X_{1}, \ldots, X_{n}$. For $n \geq 1$, define a 
centered and scaled sum

$$
Z_{n}^{*} \equiv \frac{1}{\sqrt{k_{n}}} \sum_{j=1}^{k_{n}}\left(Y_{n, j}^{*}-m_{n, \mathrm{SUB}}\right)
$$

where $m_{n, \mathrm{SUB}} \equiv \int x d S_{n, \mathrm{SUB}}(x)=N_{n}^{-1} \sum_{i=1}^{N_{n}} \tau_{b}\left[t_{n, b, i}-t_{n}\right]$ is the mean of the subsampling distribution $S_{n, \mathrm{SUB}}$, and let

$$
C_{n, k_{n}}(x) \equiv P_{*}\left(Z_{n}^{*} \leq x\right), \quad x \in \mathbb{R},
$$

denote the induced resampling distribution $P_{*}$ of $Z_{n}^{*}$. Then $C_{n, k_{n}}$ represents the $k_{n}$-fold self-convolution of the subsampling distribution $S_{n, \mathrm{SUB}}$, with appropriate centering/scaling adjustments. That is,

$$
C_{n, k_{n}}(x)=\underbrace{S_{n, \mathrm{SUB}} * S_{n, \mathrm{SUB}} * \cdots * S_{n, \mathrm{SUB}}}_{k_{n} \text { times }}\left(x \sqrt{k_{n}}+k_{n} m_{n, \mathrm{SUB}}\right), \quad x \in \mathbb{R} .
$$

We consider $C_{n, k_{n}}$ as an estimator of the distribution $F_{n}$ of $T_{n}$ and formulate general conditions under which such convolved subsampling is consistent.

As suggested earlier, such results have direct implications for block bootstrap estimation as well, because the convolved subsampling estimator $C_{n, k_{n}}$ exactly matches a block bootstrap estimator in the basic sample mean case $t_{n}\left(X_{1}, \ldots, X_{n}\right)=\bar{X}_{n}$. To illustrate, consider approximating the distribution of $T_{n}=\sqrt{n}\left(\bar{X}_{n}-\mu\right)$ where $t(P) \equiv \mu=\mathrm{E} \bar{X}_{n}$ and $\tau_{n}=\sqrt{n}$. In this setting, the block bootstrap uses an analog

$$
T_{n}^{*}=\sqrt{n_{1}}\left(\bar{X}_{n_{1}}^{*}-\mathrm{E}_{*} \bar{X}_{n_{1}}^{*}\right)
$$

based on the average $\bar{X}_{n_{1}}^{*} \equiv n_{1}^{-1} \sum_{i=1}^{n_{1}} X_{i}^{*}$ from a block bootstrap sample $X_{1}^{*}, \ldots$, $X_{n_{1}}^{*}$ of size $n_{1} \equiv k_{n} b$, which is defined by drawing $k_{n}$ blocks of length $b$, independently and with replacement, from the subsample collection $\left\{\left(X_{i}, \ldots, X_{i+b-1}\right)\right.$ : $\left.i=1, \ldots, N_{n}\right\}$ and pasting these together (where above $\mathrm{E}_{*} \bar{X}_{n_{1}}^{*}=N_{n}^{-1} \sum_{i=1}^{N_{n}} b^{-1} \times$ $\sum_{j=i}^{i+b-1} X_{j}$ denotes the bootstrap expectation of $\bar{X}_{n_{1}}^{*}$ ); see Chapter 2, Lahiri [19]. Most typically, the number of resampled blocks is taken as $k_{n}=\lfloor n / b\rfloor \rightarrow \infty$ so that the bootstrap sample recreates the approximate length $\lfloor n / b\rfloor b \approx n$ of the original sample. The bootstrap distribution of $T_{n}^{*}$ here is then equivalent to the convolved subsampling distribution $C_{n, k_{n}}$. This is because $T_{n}^{*}$ has the same resampling distribution as $Z_{n}^{*}$ in (2.4) as a sum of $k_{n}$ i.i.d. block averages $\left(Y_{n, i}^{*}-m_{n, \mathrm{SUB}}\right) / \sqrt{k_{n}}$, with each $Y_{n, i}^{*}$ drawn from $S_{n, \text { SUB }}$ in (2.1) where $t_{n}=\bar{X}_{n}$ and $\tau_{b}\left[t_{n, b, i}-t_{n}\right]=$ $\sqrt{b}\left[b^{-1} \sum_{j=i}^{i+b-1} X_{j}-\bar{X}_{n}\right], 1 \leq i \leq N_{n}$, for the sample mean case. Consequently, if convolved subsampling estimators $C_{n, k_{n}}$ are shown to be valid under weak conditions, such results entail that block bootstrap estimation is as well. In the following, we make comprehensive use of the fact that $C_{n, k_{n}}$ is always and exactly a block bootstrap estimator whenever the underlying statistic $t_{n}\left(X_{1}, \ldots, X_{n}\right)=\bar{X}_{n}$ is a sample mean; this holds true across all the various dependent data structures considered here, including cases where the usual block bootstrap from (2.5) requires modification for sample means (cf. long-range dependence in Section 4.3). 
3. Fundamental results for convolved subsampling. From (2.1) and the subsampling mean $m_{n, \mathrm{SUB}} \equiv \int x d S_{n, \mathrm{SUB}}(x)=N_{n}^{-1} \sum_{j=1}^{N_{n}} \tau_{b}\left[t_{n, b, i}-t_{n}\right]$, we have the variance of the original subsampling distribution $S_{n, \text { SUB }}$ as

$$
\hat{\sigma}_{n, \mathrm{SUB}}^{2} \equiv \int\left(x-m_{n, \mathrm{SUB}}\right)^{2} d S_{n, \mathrm{SUB}}(x)=\frac{1}{N_{n}} \sum_{j=1}^{N_{n}}\left(\tau_{b}\left[t_{n, b, i}-t_{n}\right]-m_{n, \mathrm{SUB}}\right)^{2},
$$

which estimates the asymptotic variance $\sigma^{2}$ of $T_{n}$ as in (2.2) (cf. [29]). Note that $\hat{\sigma}_{n, \mathrm{SUB}}^{2}$ is also the variance of the convolved subsampling distribution $C_{n, k_{n}}$ [i.e., the variance of the i.i.d. sum from (2.4)]. Correspondingly, $\hat{\sigma}_{n, \mathrm{SUB}}^{2}$ is then a block bootstrap variance estimator when applied to sample means.

Sections 3.1-3.3 provide basic distributional results for convolved subsampling estimators, describing when and how these have normal limits. These findings do not involve particular assumptions about the process $\left\{X_{t}\right\}$, but are instead expressed through properties of the original subsampling distribution $S_{n, \text { SUB }}$ and, specifically, convergence of the subsampling variance $\hat{\sigma}_{n, \mathrm{SUB}}^{2}$. Such subsampling properties can often be verified under weak assumptions about a process, allowing the limit behavior of convolved estimators $C_{n, k_{n}}$, and the block bootstrap, to be established under minimal conditions. Results in Section 3.1 address the important case where the original subsampling distribution $S_{n, \text { SUB }}$ has a normal limit (2.3), as is often natural when the statistic $T_{n} \stackrel{d}{\rightarrow} N\left(0, \sigma^{2}\right)$ is asymptotically normal. These findings are expected to be the most practical for establishing convolved subsampling $C_{n, k_{n}}$ estimation with normal targets (2.2). Dropping the condition that $S_{n, \text { SUB }}$ converges to a normal law but assuming convolved estimators $C_{n, k_{n}}$ are based on increasing convolution $k_{n} \rightarrow \infty$ of $S_{n, \mathrm{SUB}}$, Section 3.2 characterizes the convergence of $C_{n, k_{n}}$ to normal limits through the subsampling variance $\hat{\sigma}_{n, \mathrm{SUB}}^{2}$. In many problems involving the block bootstrap for sample means (cf. Section 4), where $T_{n}$ has a normal limit (2.2), these results provide both necessary and sufficient conditions for the validity of the block bootstrap as well as convolved subsampling generally. Finally, because convergence $\hat{\sigma}_{n, \mathrm{SUB}}^{2} \stackrel{p}{\rightarrow} \sigma^{2}$ of the subsampling variance emerges as central to the behavior of convolved estimators $C_{n, k_{n}}$, Section 3.3 develops basic results for establishing this feature.

3.1. Convolution of subsampling distributions with normal limits. Theorem 1 provides a sufficient condition for the general validity of the convolved estimator $C_{n, k_{n}}$ via fundamental subsampling quantities, $S_{n, \mathrm{SUB}}$ and $\hat{\sigma}_{n, \mathrm{SUB}}^{2}$.

THEOREM 1. Suppose (2.3) holds [i.e., $\sup _{x \in \mathbb{R}}\left|S_{n, \mathrm{SUB}}(x)-\Phi(x / \sigma)\right| \stackrel{p}{\rightarrow} 0$ ] and $\hat{\sigma}_{n, \mathrm{SUB}}^{2} \stackrel{p}{\rightarrow} \sigma^{2}>0$ as $n \rightarrow \infty$. Then

$$
\sup _{x \in \mathbb{R}}\left|C_{n, k_{n}}(x)-\Phi(x / \sigma)\right| \stackrel{p}{\rightarrow} 0 \quad \text { as } n \rightarrow \infty
$$

for any positive integer sequence $k_{n}$. 
Furthermore, when (2.2) holds additionally [i.e., $\left.T_{n} \stackrel{d}{\rightarrow} N\left(0, \sigma^{2}\right)\right]$, then $C_{n, k_{n}}$ is consistent for the distribution $F_{n}$ of $T_{n}$,

$$
\sup _{x \in \mathbb{R}}\left|C_{n, k_{n}}(x)-F_{n}(x)\right| \stackrel{p}{\rightarrow} 0 \quad \text { as } n \rightarrow \infty .
$$

To reiterate, the integer sequence $k_{n}, n \geq 1$, need not even be convergent in Theorem 1 . The consistency of the subsampling variance estimator $\hat{\sigma}_{n, \text { SUB }}^{2}$ automatically guarantees that, for any amount $k_{n}$ of convolution of $S_{n, \mathrm{SUB}}$, the convolved subsampling estimator $C_{n, k_{n}}$ will have a normal limit if the subsampling distribution $S_{n, \text { SUB }}$ does. In other words, if (2.2)-(2.3) hold so that $S_{n, \text { SUB }}$ is consistent, then $C_{n, k_{n}}$ will be as well provided $\hat{\sigma}_{n, \mathrm{SUB}}^{2} \stackrel{p}{\rightarrow} \sigma^{2}$. When the statistic $t_{n}\left(X_{1}, \ldots, X_{n}\right)=\bar{X}_{n}$ is a sample mean, then $C_{n, k_{n}}$ again denotes a block bootstrap estimator based on $k_{n}$ resampled blocks, which is thereby consistent under Theorem 1 for any sequence $k_{n}$, including the common choice $k_{n}=\lfloor n / b\rfloor \rightarrow \infty$.

Proposition 1 next characterizes the convolved subsampling estimator $C_{n, k_{n}}$ under bounded levels $k_{n}$ of convolution. In this case, a normal limit for the subsampling estimator $S_{n, \text { SUB }}$ entails the same for the convolved estimator $C_{n, k_{n}}$, provided the mean $m_{n, \mathrm{SUB}} \equiv \int x d S_{n, \mathrm{SUB}}(x)$ of the subsampling distribution converges to zero. But, if the subsampling mean $m_{n, \mathrm{SUB}}$ converges in this fashion, a normal limit for $C_{n, k_{n}}$ with bounded $\left\{k_{n}\right\}$ is equivalent to a normal limit for the original subsampling distribution $S_{n, \mathrm{SUB}}$.

Proposition 1. Suppose $\sup _{n} k_{n}<\infty$ :

(i) If (2.3) holds [i.e., $\left.\sup _{x \in \mathbb{R}}\left|S_{n, \mathrm{SUB}}(x)-\Phi(x / \sigma)\right| \stackrel{p}{\rightarrow} 0\right]$, then

$$
\sup _{x \in \mathbb{R}}\left|C_{n, k_{n}}(x)-\Phi(x / \sigma)\right| \stackrel{p}{\rightarrow} 0 \quad \text { as } n \rightarrow \infty
$$

if and only if $m_{n, \mathrm{SUB}} \equiv \int x d S_{n, \mathrm{SUB}}(x) \stackrel{p}{\rightarrow} 0$.

(ii) If $m_{n, \mathrm{SUB}} \stackrel{p}{\rightarrow} 0$ as $n \rightarrow \infty$, then (2.3) holds if and only if

$$
\sup _{x \in \mathbb{R}}\left|C_{n, k_{n}}(x)-\Phi(x / \sigma)\right| \stackrel{p}{\rightarrow} 0 \quad \text { as } n \rightarrow \infty .
$$

When the original subsampling estimator $S_{n, \text { SUB }}$ is consistent for a distribution with a normal limit [i.e., (2.2)-(2.3)], both Theorem 1 and Proposition 1 show that the convolved subsampling estimator $C_{n, k_{n}}$ is consistent under an additional subsampling moment condition. With bounded levels $k_{n}$ of convolution, the additional condition under Proposition 1 is that the subsampling mean converge $m_{n, \mathrm{SUB}} \stackrel{p}{\rightarrow} 0$. But, for general and potentially unbounded $k_{n}$, the additional condition from Theorem 1 for consistency of $C_{n, k_{n}}$ is a convergent subsampling variance $\hat{\sigma}_{n, \text { SUB }}^{2} \stackrel{p}{\rightarrow} \sigma^{2}$. With diverging amounts $k_{n} \rightarrow \infty$ of convolution, which is often encountered in 
practice and in connection to the block bootstrap, it turns out that convergence $\hat{\sigma}_{n, \mathrm{SUB}}^{2} \stackrel{p}{\rightarrow} \sigma^{2}$ is also necessary for consistency of the convolved estimator $C_{n, k_{n}}$, as treated in the next section.

3.2. Unbounded convolution of subsampling distributions. We next consider the behavior of convolved subsampling estimators with unbounded convolution $k_{n} \rightarrow \infty$ as $n \rightarrow \infty$, which arises, for example, with the block bootstrap $C_{n, k_{n}}$ for sample means with $k_{n}=\lfloor n / b\rfloor$ resampled blocks. Results here do not explicitly require convergence of the original subsampling estimator $S_{n, \text { SUB }}$ to a normal limit (2.3). While a reasonable condition in problems where the target quantity $T_{n} \stackrel{d}{\rightarrow}$ $N(0, \sigma)^{2}$ is asymptotically normal, limits for $S_{n, \text { SUB }}$ are not directly necessary for convolved estimators $C_{n, k_{n}}$ to yield normal limits from increasing convolution $k_{n}$ of $S_{n, \mathrm{SUB}}$. However, convergence of the subsampling variance $\hat{\sigma}_{n, \mathrm{SUB}}^{2}$ is crucial, as shown next.

THEOREM 2. Suppose $k_{n} \rightarrow \infty$ and $\int_{|x| \geq \sqrt{k_{n}} \epsilon} x^{2} d S_{n, \mathrm{SUB}}(x) \stackrel{p}{\rightarrow} 0$ for each $\epsilon>0$ as $n \rightarrow \infty$ :

(i) Then

$$
\sup _{x \in \mathbb{R}}\left|C_{n, k_{n}}(x)-\Phi(x / \sigma)\right| \stackrel{p}{\rightarrow} 0
$$

if and only if $\hat{\sigma}_{n, \mathrm{SUB}}^{2} \stackrel{p}{\rightarrow} \sigma^{2}>0$ as $n \rightarrow \infty$.

(ii) When $\hat{\sigma}_{n, \mathrm{SUB}}^{2} \stackrel{p}{\rightarrow} \sigma^{2}>0$ as $n \rightarrow \infty$, then $C_{n, k_{n}}$ is a consistent estimator of the distribution $F_{n}$ of $T_{n}$ if and only if $T_{n} \stackrel{d}{\rightarrow} N\left(0, \sigma^{2}\right)$ [i.e., a normal limit (2.2) for $T_{n}$ holds or $\left.\sup _{x \in \mathbb{R}}\left|F_{n}(x)-\Phi(x / \sigma)\right| \rightarrow 0\right]$.

For an unbounded sequence $k_{n} \rightarrow \infty$ of convolution (e.g., block bootstrap with $k_{n}=\lfloor n / b\rfloor$ concatenated blocks), Theorem 2 imposes no direct assumption on the convergence of the original subsampling distribution, but rather that $S_{n, \text { SUB }}$ fulfills a mild truncated second moment property. From this, the convergence of the convolved subsampling estimator $C_{n, k_{n}}$ to a normal limit is completely determined by the subsampling variance $\hat{\sigma}_{n, \mathrm{SUB}}^{2}$ under Theorem 2. Furthermore, when $\hat{\sigma}_{n, \mathrm{SUB}}^{2}$ converges, the convolved estimator $C_{n, k_{n}}$ will be valid for estimating the distribution $F_{n}$ of a target quantity $T_{n}$ having a normal limit [Theorem 2(ii)]. In cases where $T_{n}$ fails to have a normal limit, the convolved estimator $C_{n, k_{n}}$ does not apply.

The following corollary of Theorem 2 shows that a convolved estimator $C_{n, k_{n}}$ will quite generally have a normal limit, provided that the subsampling variance converges $\hat{\sigma}_{n, \mathrm{SUB}}^{2} \stackrel{p}{\rightarrow} \sigma^{2}>0$ and that some other basic feature exists for the subsampling distribution $S_{n, \mathrm{SUB}}$ or for composite statistics $\left\{\tau_{b}\left[t_{n, b, i}-t_{n}\right] \equiv\right.$ 
$\left.\tau_{b}\left[t_{b}\left(X_{i}, \ldots, X_{i-b+1}\right)-t_{n}\left(X_{1}, \ldots, X_{n}\right)\right]\right\}_{i=1}^{N_{n} \equiv n-b+1}$ defining $S_{n, \text { SUB }}$ in (2.1). Essentially, Corollary 1 entails that the truncated second moment assumption in Theorem 2 is mild in conjunction with $\hat{\sigma}_{n, \mathrm{SUB}}^{2} \stackrel{p}{\rightarrow} \sigma^{2}$.

COROLlaRY 1. Suppose one of the following conditions (C.1)-(C.4) holds:

(C.1) for some distribution $J_{0}$ with variance $\sigma^{2}>0, S_{n, \mathrm{SUB}}(x) \stackrel{p}{\rightarrow} J_{0}(x)$ as $n \rightarrow \infty$ for any continuity point $x \in \mathbb{R}$ of $J_{0}$;

(C.2) for some $\epsilon_{0}>0, N_{n}^{-1} \sum_{i=1}^{N_{n}}\left[\tau_{b}\left(t_{n, b, i}-t_{n}\right)\right]^{2+\epsilon_{0}}=O_{p}(1)$;

(C.3) the subsample-based sequence $\left\{T_{b, i}^{2} \equiv \tau_{b}^{2}\left[t_{n, b, i}-t(P)\right]^{2}: i=1, \ldots\right.$, $\left.N_{n}\right\}_{n \geq 1}$ is uniformly integrable and $T_{n} \equiv \tau_{n}\left(t_{n}-t(P)\right)=O_{p}\left(\tau_{n} / \tau_{b}\right)$;

(C.4) $\left\{X_{t}\right\}$ is stationary, $\left\{T_{n}^{2}: n \geq 1\right\}$ is uniformly integrable, and $\tau_{b} / \tau_{n}=$ $O(1)$.

Then, as $n \rightarrow \infty$,

$$
\sup _{x \in \mathbb{R}}\left|C_{n, k_{n}}(x)-\Phi(x / \sigma)\right| \stackrel{p}{\rightarrow} 0
$$

for any sequence $k_{n}$ with $\lim _{n \rightarrow \infty} k_{n}=\infty$ if and only if $\hat{\sigma}_{n, \mathrm{SUB}}^{2} \stackrel{p}{\rightarrow} \sigma^{2}>0$.

REMARK 1. For reference, note $\tau_{b} / \tau_{n} \rightarrow 0$ often holds with subsample scaling as $n \rightarrow \infty$ so that conditions $\tau_{b} / \tau_{n}=O(1)$ and $T_{n}=O_{p}\left(\tau_{n} / \tau_{b}\right)$ are mild.

Hence, if $k_{n} \rightarrow \infty$ and $\hat{\sigma}_{n, \mathrm{SUB}}^{2} \stackrel{p}{\rightarrow} \sigma^{2}$, then the convolved estimator $C_{n, k_{n}}$ will converge to a normal limit if the subsampling distribution $S_{n, \mathrm{SUB}}$ is convergent (C.1) or has an appropriate stochastically bounded moment (C.2), or if the subsampling statistics related to computing $S_{n, \text { SUB }}$ have uniformly integrable second moments (C.3)-(C.4). Condition (C.4) is a special case of (C.3) under stationarity, and corresponds to an underlying assumption of Radulović [30, 31] for examining the block bootstrap estimator $C_{n, k_{n}}$ of a sample mean with stationary, mixing processes; see also Remark 2 to follow. When restricted to Condition (C.1), the " $\Leftarrow$ " part of Corollary 1 corresponds to an initial convolved subsampling result due to Politis, Romano and Wolf [29] (Proposition 4.4.1) for unbounded convolution $k_{n} \rightarrow \infty$, which was developed for establishing the block bootstrap estimator $C_{n, k_{n}}$ for the sample mean of nonstationary data, as reconsidered here in Section 4.2. Note that, for inference with $T_{n}$ having a normal $N\left(0, \sigma^{2}\right)$ limit (2.2), Condition C. 1 in Corollary 1 is perhaps most natural and approachable by verifying convergence $S_{n, \text { SUB }}$ to a normal (2.3). In which case, the implication of Corollary 1 (involving $k_{n} \rightarrow \infty$ ) for guaranteeing that convolved subsampling and block bootstrap estimators replicate normal limits when $\hat{\sigma}_{n, \mathrm{SUB}}^{2} \stackrel{p}{\rightarrow} \sigma^{2}$ also becomes a special case of Theorem 1 (involving any $k_{n}$ ). 
REMARK 2. For block bootstrap estimation of the sample mean $T_{n}=$ $\sqrt{n}\left(\bar{X}_{n}-\mathrm{E} X_{1}\right)$ with strongly mixing, stationary processes, Radulović [30,31] provides necessary and sufficient conditions for convergence of $C_{n, k_{n}}$ (with $\left.k_{n}=\lfloor n / b\rfloor \rightarrow \infty\right)$ to a normal limit, assuming $\left\{T_{n}^{2}: n \geq 1\right\}$ is uniformly integrable. Under such assumptions, the main result there is that normal limits for both $C_{n, k_{n}}$ and $T_{n}$ are equivalent. In comparison, the necessary and sufficient conditions for normality of the block bootstrap estimator $C_{n, k_{n}}$ for a mean in Theorem 2 are perhaps more basic in that the conclusions of $[30,31]$, under the additional assumptions made there, follow from Theorem 2 (cf. Corollary 1). In this sense, Theorem 2 broadly reframes the findings in [30,31], by not involving particular process assumptions (i.e., stationarity or mixing) and applying to convolved subsampling estimators $C_{n, k_{n}}$ with general statistics and arbitrarily increasing convolution levels $k_{n} \rightarrow \infty$. Further connections to, and extensions of, the results of Radulović $[30,31]$ are made in Section 4.1 for strongly mixing processes.

3.3. Consistency of subsampling variance estimators. Theorems 1-2 demonstrate that the subsampling variance $\hat{\sigma}_{n, \mathrm{SUB}}^{2}$ plays a key role in the convergence of the convolved subsampling estimator $C_{n, k_{n}}$ generally, and of the block bootstrap for the sample mean in particular. However, convergence of the subsampling distribution $S_{n, \text { SUB }}$ itself is often much easier to directly establish under weak assumptions about the process $\left\{X_{t}\right\}$; see Politis, Romano and Wolf [29] and Section 4 to follow. This raises a further question considered next: if one knows that subsampling estimator $S_{n, \text { SUB }}$ is consistent (2.3) for a normal limit, then when will the subsampling variance $\hat{\sigma}_{n, \mathrm{SUB}}^{2}$ be convergent as well, thereby guaranteeing (from Theorem 1) that the convolved estimator $C_{n, k_{n}}$ is also consistent? As shown in Theorem 3, a general characterization is possible as well as simple sufficient conditions based on moment properties of subsample statistics (e.g., $T_{b}^{2}$ ).

For $n \geq 1$, recall $T_{n} \equiv \tau_{n}\left(t_{n}\left(X_{1}, \ldots, X_{n}\right)-t(P)\right)$ and additionally define $T_{n, i} \equiv \tau_{n}\left(t_{n}\left(X_{i}, \ldots, X_{i+n-1}\right)-t(P)\right)$ for $i \geq 1$ from the statistic applied to $\left(X_{i}, \ldots, X_{i+n-1}\right)$. Based on $N_{n} \equiv n-b+1$ subsample observations of length $1 \leq b \equiv b_{n}<n$, define a distribution function

$$
D_{n, b}(x) \equiv \frac{1}{N_{n}} \sum_{i=1}^{N_{n}} P\left(T_{b, i} \leq x\right), \quad x \in \mathbb{R},
$$

as an average of subsample-based probabilities.

THEOREM 3. Suppose (2.3) and $T_{n}=o_{p}\left(\tau_{n} / \tau_{b}\right)$ as $n \rightarrow \infty$ :

(i) Then $\hat{\sigma}_{n, \mathrm{SUB}}^{2} \stackrel{p}{\rightarrow} \sigma^{2}>0$ as $n \rightarrow \infty$ if and only if, for each $\epsilon>0$,

$$
\lim _{m \rightarrow \infty} \sup _{n \geq m} P\left(\frac{1}{N_{n}} \sum_{i=1}^{N_{n}} T_{b, i}^{2} I\left(\left|T_{b, i}\right|>m\right)>\epsilon\right)=0 .
$$


(ii) Additionally, (3.2) holds whenever $\left\{Y_{b}^{2}: b \geq 1\right\}$ is uniformly integrable, where $Y_{b}$ denotes a random variable with distribution $D_{n, b}, n \geq 1$, from (3.1) [i.e., $\left.P\left(Y_{b} \leq x\right)=D_{n, b}(x), x \in \mathbb{R}\right]$. If (2.3) and $T_{n}=o_{p}\left(\tau_{n} / \tau_{b}\right)$ hold, uniform integrability of $\left\{Y_{b}^{2}: b \geq 1\right\}$ is equivalent to $\int x^{2} d D_{n, b}(x)=N_{n}^{-1} \sum_{i=1}^{N_{n}} \mathrm{E} T_{b, i}^{2} \rightarrow$ $\sigma^{2}$ as $n \rightarrow \infty$.

(iii) (3.2) also holds whenever $\left\{X_{t}\right\}$ is stationary and $\left\{T_{b}^{2}: b \geq 1\right\}$ is uniformly integrable.

REMARK 3. As $T_{n}$ is typically tight, the assumption $T_{n}=o_{p}\left(\tau_{n} / \tau_{b}\right)$ is often satisfied by a standard condition on block length: $b \rightarrow \infty$ with $b / n+\tau_{b} / \tau_{n} \rightarrow 0$. Block conditions are not, in fact, used or required in statements of Theorems 1-3 above. However, block assumptions are usually needed to show the original subsampling estimator $S_{n, \text { SUB }}$ is convergent as in (2.3), and examples of Section 4 shall impose block length conditions for this purpose.

Theorem 3 connects convergence (2.3) of subsampling distributions $S_{n, \text { SUB }}$ to the convergence of subsampling variances $\hat{\sigma}_{n, \text { SUB }}^{2}$ in a way involving no further conditions on the process or statistic beyond mild types of uniform integrability. For example, with nonstationary processes $\left\{X_{t}\right\}$, Theorem 3(ii) converts the problem of probabilistic convergence $\hat{\sigma}_{n, \mathrm{SUB}}^{2} \stackrel{p}{\rightarrow} \sigma^{2}$ into a more approachable one of subsample-moment convergence $N_{n}^{-1} \sum_{i=1}^{N_{n}} \mathrm{E} T_{b, i}^{2} \rightarrow \sigma^{2}$. To frame another implication of Theorem 3, note that many inference problems with time series involve a stationary process $\left\{X_{t}\right\}$ and a statistic $T_{n}$ with a normal limit (2.2) such that $\left\{T_{n}^{2}: n \geq 1\right\}$, and consequently $\left\{T_{b}^{2}: b \geq 1\right\}$, is uniformly integrable; see Remark 2 . In such problems, it suffices to simply establish the consistency of the subsampling estimator $S_{n, \mathrm{SUB}}(2.3)$ and then the consistency of subsampling variance $\hat{\sigma}_{n, \text { SUB }}^{2}$ follows with no further effort [by Theorem 3(iii)] along with the consistency of the convolved subsampling estimator $C_{n, k_{n}}$ (by Theorem 1). Again, with sample means, $C_{n, k_{n}}$ is a block bootstrap distribution and $\hat{\sigma}_{n, \mathrm{SUB}}^{2}$ is a block bootstrap variance estimator, so both will be consistent in this setting by showing that $S_{n, \text { SUB }}$ is consistent. This strategy has two advantages with the block bootstrap: showing the consistency of $S_{n, \mathrm{SUB}}$ is often an easier prospect than considering either $C_{n, k_{n}}$ or $\hat{\sigma}_{n, \mathrm{SUB}}^{2}$ directly, and the consistency of $S_{n, \mathrm{SUB}}$ (and thereby the bootstrap) can typically be established under weak process assumptions.

To illustrate, Section 4 applies the basic results here for establishing the convolved subsampling estimator $C_{n, k_{n}}$, as well as the block bootstrap for sample means, under different dependence structures.

4. Applications of convolved subsampling estimation. Section 4.1 first develops consistency results for convolved subsampling estimators with strongly mixing processes and general statistics. The remaining subsections then consider convolved subsampling for the particular case of the sample mean with the goal of 
generalizing and extending results for the block bootstrap across various types of dependent data, such as nonstationary mixing time processes (Section 4.2), linear time series (Section 4.3), long-range dependent processes (Section 4.4) and spatial data (Section 4.5).

Define the strong mixing coefficient of $\left\{X_{t}\right\}$ as $\alpha(k)=\sup _{i \in \mathbb{Z}}\{\mid P(A \cap B)-$ $\left.P(A) P(B) \mid: A \in \mathcal{F}_{-\infty}^{i}, B \in \mathcal{F}_{k+i}^{\infty}\right\}, k \geq 1$, where $\mathcal{F}_{-\infty}^{i}$ and $\mathcal{F}_{k+i}^{\infty}$ respectively denote $\sigma$-algebras generated by $\left\{X_{t}: t \leq i\right\}$ and $\left\{X_{t}: t \geq k+i\right\}$ (cf. [1], Chapter 16.2). Recall $\left\{X_{t}\right\}$ is strongly mixing or $\alpha$-mixing if $\lim _{k \rightarrow \infty} \alpha(k)=0$.

4.1. Convolved subsampling for general statistics under mixing. For mixing stationary time series, Radulović [30] proved consistency of block bootstrap estimation for $T_{n}=\tau_{n}\left(t_{n}\left(X_{1}, \ldots, X_{n}\right)-t(P)\right)$ based on the sample mean $t_{n}\left(X_{1}, \ldots, X_{n}\right)=\bar{X}_{n}$ with $t(P)=\mathrm{E} X_{1}$ and $\tau_{n}=\sqrt{n}$. The assumptions made were quite weak, requiring only:

(a1) a stationary, $\alpha$-mixing process fulfilling (2.2) [i.e., $\left.T_{n} \stackrel{d}{\rightarrow} N\left(0, \sigma^{2}\right)\right]$ and block lengths $b^{-1}+b / n \rightarrow 0$ as $n \rightarrow \infty$;

(a2) uniformly integrable $\left\{T_{n}^{2}: n \geq 1\right\}$.

From results in Section 3 and the equivalence between the block bootstrap and the convolved subsampling estimator $C_{n, k_{n}}$ for the sample mean, a different perspective is possible for the bootstrap findings in Radulović [30]. Under only assumption (a1) above, the subsampling estimator $S_{n, \text { SUB }}$ is consistent [i.e., (2.3) holds] for the asymptotically normal distribution of $T_{n}=\sqrt{n}\left(\bar{X}_{n}-\mathrm{E} X_{1}\right)$ (cf. Theorem 3.2.1, [29]), implying, by Theorem 1 here, that the block bootstrap estimator $C_{n, k_{n}}$ would be consistent if the subsampling variance converges $\hat{\sigma}_{n, \mathrm{SUB}}^{2} \stackrel{p}{\rightarrow} \sigma^{2}$. But, if $S_{n, \mathrm{SUB}}$ is consistent for a normal limit by (a1), assumption (a2) then guarantees that $\hat{\sigma}_{n, \mathrm{SUB}}^{2} \stackrel{p}{\rightarrow} \sigma^{2}$ holds by Theorem 3 . Furthermore, under (a2) and with $k_{n}=\lfloor n / b\rfloor \rightarrow \infty$ resampled blocks as in Radulović [30, 31], convergence $\hat{\sigma}_{n, \mathrm{SUB}}^{2} \stackrel{p}{\rightarrow} \sigma^{2}$ becomes even necessary here by Theorem 2 . Hence, $\alpha$-mixing serves to show that the original subsampling estimator $S_{n, \mathrm{SUB}}$ is consistent; after which, uniform integrability and stationary assure both $\hat{\sigma}_{n, \mathrm{SUB}}^{2} \stackrel{p}{\rightarrow} \sigma^{2}$ and consistency of the block bootstrap estimator $C_{n, k_{n}}$ by Theorems $2-3$.

Under analogously weak assumptions as those of Radulović [30], Theorem 4 next provides the general consistency of convolved subsampling estimation for general statistics arising from mixing, and possibly nonstationary, time processes. When applied to a sample mean $t_{n}\left(X_{1}, \ldots, X_{n}\right)=\bar{X}_{n}$, so that $C_{n, k_{n}}$ is a block bootstrap estimator, this result extends those of Radulović [30] in two ways: by allowing potential nonstationarity series and by permitting arbitrary levels $k_{n}$ of convolution/block resampling (rather than the single choice $k_{n}=\lfloor n / b\rfloor$ ). When the statistic $t_{n}\left(X_{1}, \ldots, X_{n}\right)$ is not a sample mean, $C_{n, k_{n}}$ may not again match the block bootstrap but can have interest as an alternative block resampling estimator (cf. Section 5). 
THEOREM 4. Let $\left\{X_{t}\right\}$ be a (possibly nonstationary) strongly mixing sequence. Suppose $b^{-1}+b / n+\tau_{b} / \tau_{n} \rightarrow 0$ as $n \rightarrow \infty ; T_{n}=o_{p}\left(\tau_{n} / \tau_{b}\right)$; (3.2) holds; and that $Y_{b} \stackrel{d}{\rightarrow} N\left(0, \sigma^{2}\right)$ as $n \rightarrow \infty$, for some $\sigma^{2}>0$, where each random variable $Y_{b}, b \equiv b_{n} \geq 1$, has distribution function $D_{n, b}$ from (3.1). Then, as $n \rightarrow \infty$,

$$
\sup _{x \in \mathbb{R}}\left|S_{n, \mathrm{SUB}}(x)-\Phi(x / \sigma)\right| \stackrel{p}{\rightarrow} 0 \quad \text { and } \quad \hat{\sigma}_{n, \mathrm{SUB}}^{2} \stackrel{p}{\rightarrow} \sigma^{2}
$$

and, for any positive integer sequence $k_{n}$,

$$
\sup _{x \in \mathbb{R}}\left|C_{n, k_{n}}(x)-\Phi(x / \sigma)\right| \stackrel{p}{\rightarrow} 0 .
$$

Furthermore, if (2.2) additionally holds [i.e., $\left.T_{n} \stackrel{d}{\rightarrow} N\left(0, \sigma^{2}\right)\right]$, then $S_{n, \mathrm{SUB}}$ and $C_{n, k_{n}}$ (with any $k_{n}$ ) are consistent for the distribution $F_{n}$ of $T_{n}$ :

$$
\sup _{x \in \mathbb{R}}\left|S_{n, \operatorname{SUB}}(x)-F_{n}(x)\right| \stackrel{p}{\rightarrow} 0 \quad \text { and } \quad \sup _{x \in \mathbb{R}}\left|C_{n, k_{n}}(x)-F_{n}(x)\right| \stackrel{p}{\rightarrow} 0 .
$$

While providing a broad result on the validity of convolved subsampling estimation for mixing processes, Theorem 4 also expands the general subsampling results of Politis, Romano and Wolf [29] (Chapter 4.2), which focused on $S_{n, \text { SUB }}$ for mixing series, to further include consistency of the subsampling variance $\hat{\sigma}_{n, \text { SUB }}^{2}$. That is, when dropping (3.2), the remaining Theorem 4 assumptions are minimal and match those of Theorem 3.2.1-4.2.1 of Politis, Romano and Wolf [29] for the consistency of $S_{n}$,SUB to a normal limit; including (3.2) in Theorem 4 is then necessary for $\hat{\sigma}_{n, \mathrm{SUB}}^{2} \stackrel{p}{\rightarrow} \sigma^{2}$ by Theorem 3 and assures convergence of $C_{n, k_{n}}$ by Theorem 1 .

If the process $\left\{X_{t}\right\}$ is actually stationary, we immediately obtain the following result.

COROLlARY 2. Let $\left\{X_{t}\right\}$ be a stationary, strongly mixing sequence. Suppose also $b^{-1}+b / n+\tau_{b} / \tau_{n} \rightarrow 0$ as $n \rightarrow \infty$; that (2.2) holds; and that (3.2) holds (e.g., uniform integrability of $\left\{T_{n}^{2}: n \geq 1\right\}$ suffices). Then, as $n \rightarrow \infty$, the convergence results of Theorem 4 hold.

Section 5 illustrates Theorem 4 for establishing convolved subsampling with mixing time series and several general classes of statistics. These represent cases where $C_{n, k_{n}}$ differs from the block bootstrap estimator.

However, Section 4.2 first provides some further refinements with mixing processes in the sample mean case, where $C_{n, k_{n}}$ matches the block bootstrap.

4.2. Block bootstrap for mixing nonstationary time processes. Consider a strongly mixing, potentially nonstationary sequence $\left\{X_{t}\right\}$ having a common mean parameter $\mathrm{E} X_{t}=\mu \in \mathbb{R}$, which is estimated by the sample mean $\bar{X}_{n}$. In this setting and under conditions where $T_{n} \equiv \sqrt{n}\left(\bar{X}_{n}-\mu\right)$ has a normal limit (2.2), 
Fitzenberger [12] established the consistency of the block bootstrap for estimating the distribution of $T_{n}$. The result, however, required the existence of a $(4+\delta)$ moment (i.e., $\sup _{t} \mathrm{E}\left|X_{t}\right|^{4+\delta}<\infty$ for some $\delta>0$ ) along with stringent mixing conditions and restrictions on the block length $b=o\left(n^{1 / 2}\right)$. Politis, Romano and Wolf [29] (Example 4.4.1) showed that the subsampling estimator $S_{n, \text { SUB }}$ is consistent under weaker conditions, including only a $(2+\delta)$-moment. For the block bootstrap with $k_{n}=\lfloor n / b\rfloor$ resampled blocks, Politis, Romano and Wolf [29] also proved bootstrap consistency by applying convolved subsampling in this problem, using a weaker block assumption $b=o(n)$ than Fitzenberger [12] but otherwise with same remaining strong assumptions about the process. However, [29] (Remark 4.4.4) conjectured that the block bootstrap might be established under nonstationarity using the same weak moment/mixing conditions as the subsampling estimator $S_{n, \mathrm{SUB}}$, just as in the case of stationary mixing processes (cf. [30]). We confirm this by the following Theorem 5 .

THEOREM 5. Let $\left\{X_{t}\right\}$ be a sequence of (not necessarily stationary) strongly mixing random variables with common mean $\mu$. For some $\delta>0$, suppose that $\sup _{t} \mathrm{E}\left|X_{t}\right|^{2+\delta}<\infty$ and $\sum_{k=1}^{\infty} \alpha(k)^{\delta /(2+\delta)}<\infty$. Assume also that, for some $\sigma^{2}>$ 0 ,

$$
\lim _{n \rightarrow \infty} \sup _{i \geq 1}\left|\operatorname{Var}\left(n^{-1 / 2} \sum_{t=i}^{i+n-1} X_{t}\right)-\sigma^{2}\right|=0 .
$$

Then, as $n \rightarrow \infty, T_{n}=\sqrt{n}\left(\bar{X}_{n}-\mu\right) \stackrel{d}{\rightarrow} N\left(0, \sigma^{2}\right)$ [i.e., (2.2) holds]. Additionally, if $b^{-1}+b / n \rightarrow 0$ as $n \rightarrow \infty$, then

$$
\sup _{x \in \mathbb{R}}\left|S_{n, \mathrm{SUB}}(x)-\Phi(x / \sigma)\right| \stackrel{p}{\rightarrow} 0 \quad \text { and } \quad \hat{\sigma}_{n, \mathrm{SUB}}^{2} \stackrel{p}{\rightarrow} \sigma^{2}
$$

and, for any positive integer sequence $k_{n}$,

$$
\sup _{x \in \mathbb{R}}\left|C_{n, k_{n}}(x)-\Phi(x / \sigma)\right| \stackrel{p}{\rightarrow} 0 .
$$

Hence, with any number $k_{n}$ of concatenated blocks, the block bootstrap estimator $C_{n, k_{n}}$ is valid for the distribution of the sample mean under mild assumptions for mixing, and possibly nonstationary processes. Note that the assumptions of Theorem 5 resemble those essentially needed for a central limit theorem (CLT) itself (cf. Theorem 16.3.5, [1]). In particular, the assumptions also match those commonly used in the stationary case for establishing the block bootstrap; see Section 3.2 of Lahiri [19]. With the same moment condition as Politis, Romano and Wolf [29] (Theorem 4.4.1), Theorem 5 additionally shows that the original subsampling estimator $S_{n, \mathrm{SUB}}$ is consistent under nonstationarity with even weaker mixing assumptions than considered previously $\sum_{k=1}^{\infty}(k+1)^{2} \alpha(k)^{\delta /(8+\delta)}<\infty$. The central message of Theorem 5, however, is that the convolved subsampling 
approach allows the block bootstrap estimator $C_{n, k_{n}}$ for the sample mean to be established under weak conditions similarly to $S_{n, \text { SUB }}$.

Next, consider the block bootstrap in another important example of nonstationarity, involving certain periodically correlated time series. Here, the mean function $\mu(t) \equiv \mathrm{E} X_{t}$ is not constant, as in Theorem 5, but rather an almost periodic function. A real-valued function $f$ is almost periodic if, for every $\epsilon>0$, there is an $n(\epsilon) \in \mathbb{N}$ such that in every interval $I_{n(\epsilon)}$ of length $n(\epsilon)$ or greater, there is an integer $p \in I_{n(\epsilon)}$ such that

$$
\sup _{t \in \mathbb{Z}}|f(t+p)-f(t)|<\epsilon ;
$$

see [7]. For such functions, the limit $M(f) \equiv \lim _{n \rightarrow \infty} n^{-1} \sum_{i=s}^{s+n-1} f(i)$ exists and does not depend on $s$. Moreover, if the set $\Lambda=\left\{\lambda \in[0,2 \pi): M\left(g_{\lambda}\right) \neq 0\right\}$ is finite for $g_{\lambda}(t) \equiv f(t) e^{-l \lambda t}, t \in \mathbb{Z}(l=\sqrt{-1})$, then

$$
\left|\frac{1}{n} \sum_{i=s}^{s+n-1}(f(i)-M(f))\right| \leq \frac{C}{n}
$$

holds for some $C>0$ not depending on $n$ or $s$ by Cambanis et al. [6]. Hence, $M(f)$ represents the mean value of an almost periodic function $f$. A time series is called almost periodically correlated (APC) if its mean and autocovariance functions are almost periodic, that is, for every fixed $\tau \in \mathbb{Z}$,

$$
\mu(t)=\mathrm{E} X_{t} \quad \text { and } \quad \rho_{\tau}(t)=\mathrm{E} X_{t} X_{t+\tau}
$$

are almost periodic as functions of $t$; see [14]. For an ACP series $\left\{X_{t}\right\}$, a parameter of interest is then $t(P) \equiv M(\mu)=\lim _{n \rightarrow \infty} n^{-1} \sum_{i=s}^{s+n-1} \mu(i)$ as a summary of the process mean structure, which is estimated by $\bar{X}_{n}$. Synowiecki [34] showed that the block bootstrap consistently estimates the sampling distribution of $T_{n}=n^{1 / 2}\left(\bar{X}_{n}-M(\mu)\right)$ under appropriate conditions. By applying the convolved subsampling technique, we may extend the bootstrap results of Synowiecki [34] (Corollary 3.2) by substantially weakening the assumptions made there about $(4+\delta)$-moments and $\sum_{k=1}^{\infty} k \alpha(k)^{\delta /(4+\delta)}<\infty$.

COROLLARY 3. Let $\left\{X_{t}\right\}$ be an APC sequence of strongly mixing random variables such that $\sup _{t} \mathrm{E}\left|X_{t}\right|^{2+\delta}<\infty$ and $\sum_{k=1}^{\infty} \alpha(k)^{\delta /(\delta+2)}<\infty$ for some $\delta>0$, and suppose the set $\Lambda=\left\{\lambda \in[0,2 \pi): M\left(g_{\lambda}\right) \neq 0\right\}$ is finite for $g_{\lambda}(t) \equiv$ $\mu(t) e^{-\imath \lambda t}, t \in \mathbb{Z}$, with $\mu(t)=\mathrm{E} X_{t}$. Then all conclusions of Theorem 5 hold for $T_{n}=n^{1 / 2}\left(\bar{X}_{n}-M(\mu)\right)$ as $n \rightarrow \infty$.

4.3. Block bootstrap for linear time processes. Based on a sample $X_{1}, \ldots, X_{n}$, next consider inference about the mean $\mathrm{E} X_{t}=\mu \in \mathbb{R}$ of a stationary time process $\left\{X_{t}\right\}$ prescribed as

$$
X_{t}=\mu+\sum_{j \in \mathbb{Z}} a_{j} \varepsilon_{t-j}, \quad t \in \mathbb{Z},
$$


in terms of i.i.d. variables $\left\{\varepsilon_{t}\right\}$ with mean zero and finite variance $\mathrm{E} \varepsilon_{t}^{2} \in(0, \infty)$ and a real-valued sequence $\left\{a_{j}\right\}$ of constants where $\sum_{j \in \mathbb{Z}} a_{j}^{2}<\infty$. The linear series $\left\{X_{t}\right\}$ need not be mixing and, depending on constants $\left\{a_{j}\right\}$, can potentially exhibit either weak or strong forms of time dependence. Using the sample mean $\bar{X}_{n}$ to estimate the process mean $\mu$, suppose that

$$
\lim _{n \rightarrow \infty} n^{\alpha} \operatorname{Var}\left(\bar{X}_{n}\right)=\sigma^{2}
$$

for some $\sigma^{2}>0$ and exponent $\alpha \in(0,1]$ depending on the process $\left\{X_{t}\right\}$. When $\alpha=1$, the sample mean's variance decays at a rate $O\left(n^{-1}\right)$ with the sample size, as typical for weakly, or short-range, dependent processes. However, when $\alpha \in$ $(0,1)$, the sample mean has a variance with comparatively slower decay $O\left(n^{-\alpha}\right)$, which may be associated with processes exhibiting strong or long-range forms of dependence. Long-range dependent processes are commonly characterized by slowly decaying covariances involving a long-memory exponent $\alpha \in(0,1)$, which results in less precision (4.3) for a sample mean compared to the weak dependence case [3]. Classes of strongly dependent processes that satisfy (4.2)-(4.3) include fractional Gaussian models [26] and fractional autoregressive integrated moving averages [13].

Based on (4.3), define $T_{n} \equiv n^{\alpha / 2}\left(\bar{X}_{n}-\mu\right)$ in terms of scaling $\tau_{n} \equiv n^{\alpha / 2}$. In this setting, the convolved subsampling $C_{n, k_{n}}$ once again corresponds to the block bootstrap estimator based on $k_{n}$ resampled blocks, but there is a wrinkle to note. Recalling from (2.5) that the bootstrap sample mean $\bar{X}_{n 1}^{*}$ is created from a bootstrap sample of length $n_{1}=k_{n} b$, the bootstrap rendition of $T_{n}$ here is given by

$$
T_{n}^{*} \equiv b^{(1-\alpha) / 2}\left(n_{1}\right)^{\alpha / 2}\left(\bar{X}_{n_{1}}^{*}-\mathrm{E}_{*} \bar{X}_{n_{1}}^{*}\right)
$$

rather than the analog $T_{n}^{*}=\left(n_{1}\right)^{\alpha / 2}\left(\bar{X}_{n_{1}}^{*}-\mathrm{E}_{*} \bar{X}_{n_{1}}^{*}\right)$ of (2.5). While intuitive, the latter is incorrect under long memory and produces a degenerate bootstrap [18]. Instead, the bootstrap from (4.4) requires an adjustment $b^{(1-\alpha) / 2}$, which disappears under weak dependence $\alpha=1$ whereby bootstrap versions of $T_{n}$ then match in (2.5) and (4.4). Interestingly, convolved subsampling estimator $C_{n, k_{n}}$ automatically corresponds to the correct bootstrap rendition $T_{n}^{*}$ in (4.4) under both weak $\alpha=1$ and strong $\alpha \in(0,1)$ dependence.

Considering the sample mean from stationary linear processes (4.2) ranging over short- or long-range dependence, Kim and Nordman [16] showed the consistency of the block bootstrap distribution $C_{n, k_{n}}$ (when $k_{n}=\lfloor n / b\rfloor$ ) and bootstrap variance $\hat{\sigma}_{n, \mathrm{SUB}}^{2}$. Via convolved subsampling, we may generalize their results. For linear processes $\left\{X_{t}\right\}$ satisfying (4.2)-(4.3), the sample mean $T_{n} \equiv n^{\alpha / 2}\left(\bar{X}_{n}-\mu\right)$ has a normal limit (2.2) (cf. [8]) and the subsampling estimator $S_{n, \text { SUB }}$ is also consistent [i.e., (2.3) holds] under mild assumptions (cf. [27]). Hence, by primitively assuming (2.2)-(2.3) to hold, Corollary 4 next extends the block bootstrap to general stationary processes with sample means satisfying a variance condition (4.3), which includes results of Kim and Nordman [16] for linear processes as a special case. 
COROLLARY 4. Let $\left\{X_{t}\right\}$ be a stationary process with mean $\mu \in \mathbb{R}$ satisfying (4.3) for some $\alpha \in(0,1]$, and suppose that (2.2)-(2.3) hold for $T_{n} \equiv n^{\alpha / 2}\left(\bar{X}_{n}-\mu\right)$. Then, as $n \rightarrow \infty$,

$$
\hat{\sigma}_{n, \mathrm{SUB}}^{2} \stackrel{p}{\rightarrow} \sigma^{2} \quad \text { and } \quad \sup _{x \in \mathbb{R}}\left|C_{n, k_{n}}(x)-\Phi(x / \sigma)\right| \stackrel{p}{\rightarrow} 0
$$

for any positive integer sequence $k_{n}$.

Corollary 4 is an application of Theorems 1 and 3 for stationary processes which may not be strongly mixing. Our exposition has assumed the exponent $\alpha \in(0,1]$ to be known. Upon replacing $\alpha$ with an estimator $\hat{\alpha} \equiv \hat{\alpha}\left(X_{1}, \ldots, X_{n}\right)$ where $\mid \hat{\alpha}-$ $\alpha \mid \log n \stackrel{p}{\rightarrow} 0$, the conclusions of Corollary 4 still hold; see Remark 3 of [16] for further details.

4.4. Block bootstrap under long-range dependence. This section briefly mentions the block bootstrap with additional types of long-memory sequences. Beyond linear processes, the sample mean of a long-range dependent sequence may converge to a nonnormal limit, such as the case for certain subordinated Gaussian processes considered by Taqqu [35] and Dobrushin and Major [10] [e.g., $X_{t}=G\left(Z_{t}\right)$ as a function $G$ of a long-range dependent Gaussian series $\left.\left\{Z_{t}\right\}\right]$. For such time series, Lahiri [18] proved that the block bootstrap sample mean always has a normal limit, so that the block bootstrap fails if the original sample mean is asymptotically nonnormal. This result is in concordance with our Theorem 2(ii).

Zhang et al. [36] considered subsampling for a wider class of long-memory series that includes both subordinated Gaussian processes as well types of linear processes (4.2). Namely, sequences $X_{t}=K\left(Z_{t}\right), t \in \mathbb{Z}$, formed by a measurable transformation $K$ of a long-range dependent linear process

$$
Z_{t}=\varepsilon_{t}+\sum_{j=1}^{\infty} j^{-\beta} L(j) \varepsilon_{t-j}, \quad t \in \mathbb{Z},
$$

defined with i.i.d. mean zero, finite variance innovations $\left\{\varepsilon_{t}\right\}$, an index parameter $1 / 2<\beta<1$ and slowly varying function $L(\cdot)$. They distinguish two cases, depending on $\beta$ and the so-called power rank $p \geq 1$ of $K$. In the first case [i.e., $p(2 \beta-1)>1]$, the transformation $K$ diminishes long-range dependence, and the sample mean converges to a normal limit. In the second case [i.e., $p(2 \beta-1)<1$ ], the transformed process $X_{t}=K\left(Z_{t}\right)$ remains strongly dependent and the sample mean has a normal limit only when $p=1$.

Assuming a constant function $L(\cdot)=C$ in the above formulation, the variance of a sample mean satisfies (4.3) [i.e., $\lim _{n \rightarrow \infty} n^{\alpha} \operatorname{Var}\left(\bar{X}_{n}\right)=\sigma^{2}>0$ ] with a longmemory exponent $\alpha \equiv \min \{1, p(2 \beta-1)\} \in(0,1]$ that changes between cases of weak $\alpha=1$ or strong $\alpha=p(2 \beta-1) \in(0,1)$ dependence (cf. Lemma 1, [36]). For the sample mean, Zhang et al. [36] established consistency of several subsampling 
estimators as well as convergence of $\hat{\sigma}_{n, \mathrm{SUB}}^{2}$. Thus, by slightly recasting results of [36] and applying our Corollary 4, we may show the validity of the block bootstrap $C_{n, k_{n}}$ for estimating the distribution of $T_{n} \equiv n^{\alpha / 2}\left(\bar{X}_{n}-\mu\right), \mu=\mathrm{E} X_{t}$, for transformed linear processes exhibiting either short- or long-range dependence. To the best of our knowledge, the bootstrap has not yet been investigated for such processes.

COROLlary 5. For $X_{t}=K\left(Z_{t}\right), t \in \mathbb{Z}$, as above, suppose (4.3) holds for $\alpha=$ $\min \{1, p(2 \beta-1)\} \in(0,1]$ along with conditions of Theorem 1 in [36] [involving $a$ block $b \propto n^{a}$ for some $\left.a \in(0,1)\right]$ with either $p(2 \beta-1)>1$, or $p=1$ and $(2 \beta-1)<1$. Then, for $T_{n}=n^{\alpha / 2}\left(\bar{X}_{n}-\mu\right)$ as $n \rightarrow \infty$, both (2.2)-(2.3) hold and

$$
\hat{\sigma}_{n, \mathrm{SUB}}^{2} \stackrel{p}{\rightarrow} \sigma^{2} \quad \text { and } \quad \sup _{x \in \mathbb{R}}\left|C_{n, k_{n}}(x)-\Phi(x / \sigma)\right| \stackrel{p}{\rightarrow} 0
$$

for any positive integer sequence $k_{n}$.

As with subordinated Gaussian processes [18], consistency of the block bootstrap or convolved estimator $C_{n, k_{n}}$ for the sample mean only follows in cases where a CLT holds. For subordinated Gaussian processes and statistics other than the sample mean, Betken and Wendler [4] proved the general consistency of the subsampling estimator, and Bai and Taqqu [2] established weak conditions for the subsample size. When the original statistic has a normal limit, consistency of convolved subsampling will follow by our Theorem 2 by showing convergence of $\hat{\sigma}_{n, \mathrm{SUB}}^{2}$ (which, as [2] and [4] consider stationary processes, can hold by Theorem 3 and uniform integrability).

4.5. Spatial data. While convolved subsampling results have been presented for processes $\left\{X_{t}\right\}$ indexed by time $t$ to ease the exposition, Theorems 1-3 also apply to more general processes, including spatial random fields. In the Supplementary Material [15], we illustrate this with spatial data on a grid, for which various authors have considered block bootstrap and subsampling; see Lahiri [19] (Chapter 12) and Politis, Romano and Wolf [29] (Chapter 5) and references therein. Under appropriate assumptions for the stationary random field, the spatial sample mean has a normal limit and we establish convolved subsampling under mixing conditions from Lahiri [20] (Section 4.2) which are almost optimal, or minimal, for a spatial CLT. The result given also demonstrates the spatial block bootstrap for the sample mean under weaker mixing/moment conditions than considered previously (cf. Theorem 12.1, [19]).

5. Convolved subsampling in other contexts. We briefly outline relationships between convolved subsampling and some recent literature about block resampling for statistics outside of the sample mean cases in Sections 4.2-4.5. As alternatives to bootstrap, such works have considered generalized approaches to resampling that are convolved subsampling. When viewed as such, these previous 
developments may be unified and simplified by general results here for mixing time series (or processes) (cf. Section 4.1), as illustrated in Section 5.1 for Ustatistics and Section 5.2 for spectral estimators. Section 5.3 mentions extensions to further statistics, such as L-estimators.

For the classes of statistics next considered, our results with convolved subsampling cannot be used to directly justify the block bootstrap. However, our findings may still contribute to this end, as explained in Section 8.

5.1. U-statistics. U-statistics are a class of nonlinear functionals for prescribing statistics, such as the sample variance. Suppose that $X_{1}, \ldots, X_{n}$ arise from a stationary process and, based on a symmetric kernel $h: \mathbb{R}^{2} \rightarrow \mathbb{R}$, define a (bivariate) U-statistic as

$$
t_{n} \equiv t_{n}\left(X_{1}, \ldots, X_{n}\right)=\frac{2}{n(n-1)} \sum_{1 \leq i<j \leq n} h\left(X_{i}, X_{j}\right),
$$

which estimates a target parameter $t(P) \equiv \int h(x, y) d G(x) d G(y)$, where $G$ denotes the marginal distribution of $X_{t}$. Consider the problem of estimating the distribution of $T_{n} \equiv \sqrt{n}\left(t_{n}-t(P)\right)$, with scaling $\tau_{n}=\sqrt{n}$, under weak time dependence. The subsampling distribution $S_{n, \mathrm{SUB}}$ is defined by computing the Ustatistic $t_{n, b, i}=[b(b-1)]^{-1} 2 \sum_{i \leq j_{1}<j_{2} \leq i+b-1} h\left(X_{j_{1}}, X_{j_{2}}\right)$ on each length $b$ subsample $\left\{\left(X_{i}, \ldots, X_{i+b-1}\right)\right\}_{i=1}^{N_{n} \equiv n-b+1}$ in (2.1). In contrast, block bootstrap versions of U-statistics have a formulation similar to (2.5); see Dehling and Wendler [9], Sharipov and Wendler [33] and Leucht [23]. That is, a bootstrap sample $X_{1}^{*}, \ldots, X_{n_{1}}^{*}, n_{1}=k_{n} b$, is generated by resampling $k_{n}$ blocks of length $b$ (typically $\left.k_{n}=\lfloor n / b\rfloor\right)$ and then the U-statistic $t_{n_{1}}^{*} \equiv t_{n_{1}}\left(X_{1}^{*}, \ldots, X_{n_{1}}^{*}\right)$ is calculated from the complete bootstrap sample to create a bootstrap rendition $T_{n}^{*}=\sqrt{n_{1}}\left(t_{n_{1}}^{*}-\mathrm{E}_{*} t_{n_{1}}^{*}\right)$ of $T_{n}$. In this setting, the bootstrap distribution $T_{n}^{*}$ would not generally correspond to that of a $k_{n}$-fold convolution $C_{n, k_{n}}$ of the subsampling distribution $S_{n, \mathrm{SUB}}$, as occurred in the sample mean case (Section 2.2).

However, Sharipov, Tewes and Wendler [32] recently considered an alternative block resampling estimator for U-statistics, which matches the convolved subsampling estimator $C_{n, k_{n}}$ here based on the subsampling estimator $S_{n, \text { SUB }}$ for $T_{n}$ described above. Note that, for stationary mixing data, Dehling and Wendler [9] (Theorem 1.8-Lemma 3.6) provide a CLT for the relevant U-statistic: $T_{n} \stackrel{d}{\rightarrow}$ $N\left(0, \sigma^{2}\right)$ and $\mathrm{E} T_{n}^{2} \rightarrow \sigma^{2}$ as $n \rightarrow \infty$ where $\sigma^{2} \equiv 4 \sum_{k=-\infty}^{\infty} \operatorname{Cov}\left(h_{1}\left(X_{0}\right), h_{1}\left(X_{k}\right)\right)$ for $h_{1}(x)=\int h(x, y) d G(y)$. Under mixing conditions and with $k_{n}=\lfloor n / b\rfloor \rightarrow$ $\infty$, Sharipov, Tewes and Wendler [32] established that $C_{n, k_{n}}$ captures this limiting normal distribution of $T_{n}$ and also showed the consistency of the variance $\hat{\sigma}_{n, \text { SUB }}^{2}$ of $C_{n, k_{n}}$. The argument there involved decomposing the bootstrap U-statistic $T_{n}^{*}$ into a linear part, coinciding with a sample mean from the usual block bootstrap, and degenerate part shown to be negligible. However, the general convolution result in Theorem 4 for mixing processes provides an alternative, and much simpler, 
approach. From $T_{n} \stackrel{d}{\rightarrow} N\left(0, \sigma^{2}\right)$ and $\mathrm{E} T_{n}^{2} \rightarrow \sigma^{2}$, all of the conditions of Theorem 4 automatically hold, proving that $C_{n, k_{n}}$ is consistent for the distribution of $T_{n}$ for any convolution level $k_{n}$ and also that $\hat{\sigma}_{n, \mathrm{SUB}}^{2} \stackrel{p}{\rightarrow} \sigma^{2}$. This approach also weakens the block assumptions used by [32] [i.e., $b=O\left(n^{\epsilon}\right)$ for some $\epsilon \in(0,1)$ ] to $b^{-1}+b / n \rightarrow 0$ under Theorem 4 .

5.2. Spectral estimators for nonstationary time series. As described in Section 4.2, almost periodically correlated (APC) time series $\left\{X_{t}\right\}$ are an important example of nonstationary sequences. Beyond the mean function, inference about the correlation structure is also of interest. Based on a sample $X_{1}, \ldots, X_{n}$, a symmetric kernel $w(\cdot)$ and a bandwidth choice $L_{n}$, Lenart [21, 22] considered kernel estimators

$$
t_{n}\left(X_{1}, \ldots, X_{n}\right) \equiv \frac{1}{2 \pi n} \sum_{t=1}^{n} \sum_{s=1}^{n} \frac{1}{L_{n}} w\left(\frac{t-s}{L_{n}}\right) X_{t} X_{s} e^{-\imath v t} e^{\imath \omega s}
$$

for an extended spectral density $t(P) \equiv t(P)(v, \omega),(v, \omega) \in(0,2 \pi]^{2}$, used to represent the almost periodic covariance function $c_{\tau}(t) \equiv \operatorname{Cov}\left(X_{t}, X_{t+\tau}\right), t \in \mathbb{Z}$, for a given $\tau \in \mathbb{Z}$; see $[21,22]$ for details.

For $T_{n} \equiv \tau_{n}\left(t_{n}-t(P)\right)$ with scaling $\tau_{n}=\sqrt{n / L_{n}}$, Lenart [21] (Theorems 3.13.2) proved a CLT $T_{n} \stackrel{d}{\rightarrow} N\left(0, \sigma^{2}\right)$ and moment convergence $\mathrm{E} T_{n}^{2} \rightarrow \sigma^{2}$ with mixing APC series, which was extended in Lenart [22] to multivariate data. Due to the complicated form of $\sigma^{2}$, a subsampling estimator $S_{n, \mathrm{SUB}}$ for the distribution of $T_{n}$ may computed as in (2.1) with analog statistics $t_{n, b, i}$ and scaling $\tau_{b}=\sqrt{b / L_{b}}$ defined from subsamples $\left\{\left(X_{i}, \ldots, X_{i+b-1}\right)\right\}_{i=1}^{N_{n} \equiv n-b+1}$. Lenart [21] proved the consistency of the estimator $S_{n, \mathrm{SUB}}$, while Lenart [22] proposed a generalized resampling method which essentially corresponds to a convolved subsampling estimator $C_{n, k_{n}}$ induced from $S_{n, \mathrm{SUB}}$. In particular, Lenart [22] established the consistency of $C_{n, k_{n}}$ through bootstrap arguments requiring much stronger mixing and moment assumptions than needed for the convergence $T_{n} \stackrel{d}{\rightarrow} N\left(0, \sigma^{2}\right)$ and $\mathrm{E} T_{n}^{2} \rightarrow \sigma^{2}$. However, the general convolved subsampling result in Theorem 4 may alternatively be used here with mixing nonstationary ACP series.

To apply Theorem 4 with blocks where $b^{-1}+b / n+\tau_{b} / \tau_{n} \rightarrow 0$ as $n \rightarrow \infty$, one requires that $Y_{b} \stackrel{d}{\rightarrow} N\left(0, \sigma^{2}\right)$ and that (3.2) holds, where $Y_{b}, b \equiv b_{n} \geq 1$, denotes a sequence of variables with distribution $D_{n, b}(\cdot)$ from (3.1). But, the same conditions needed for $T_{n} \stackrel{d}{\rightarrow} N\left(0, \sigma^{2}\right)$ and $\mathrm{E} T_{n}^{2} \rightarrow \sigma^{2}$ also yield $Y_{b} \stackrel{d}{\rightarrow} N\left(0, \sigma^{2}\right)$ and $\mathrm{E} Y_{b}^{2} \rightarrow \sigma^{2}$ (cf. Theorems 3.1-3.2 and 4.1, [21]). Furthermore, mixing and $Y_{b} \stackrel{d}{\rightarrow} N\left(0, \sigma^{2}\right)$, along with $T_{n}=O_{p}(1)$ and $\tau_{n} / \tau_{b} \rightarrow \infty$, guarantee that $(2.3)$ holds [i.e., $\sup _{x \in \mathbb{R}}\left|S_{n, \mathrm{SUB}}(x)-\Phi(x / \sigma)\right| \stackrel{p}{\rightarrow} 0$ ] and that consequently (3.2) follows from Theorem 3(ii) by $\mathrm{EY}_{b}^{2} \rightarrow \sigma^{2}$. That is, the same minimal conditions for a CLT with APC series suffice for the consistency of convolved subsampling $C_{n, k_{n}}$ by the general result of Theorem 4 . 
5.3. L-estimators and other statistics beyond the sample mean. Other classes of statistics with normal limits, where resampling may be helpful, include Mestimators, L-statistics and generalizations such as GL-statistics. Just as for the U-statistics and spectral estimators in the previous sections, convolved subsampling may be applied as a resampling method which is neither classical subsampling nor the block bootstrap for general statistics. As an advantage in such cases, convolved subsampling is often verifiable under mild assumptions (cf. Sections 3, 4.1), which we mention for L-estimators. If $X_{1}, \ldots, X_{n}$ denotes a stationary stretch with marginal quantile function $G^{-1}$, an L-estimator $t_{n}=\int_{0}^{1} \hat{G}_{n}^{-1}(u) J(u) d u$ of a parameter $t(P)=\int_{0}^{1} G^{-1}(u) J(u) d u$ represents a linear combinations of order statistics, defined by the quantiles of the empirical distribution $\hat{G}_{n}(x)=$ $n^{-1} \sum_{t=1}^{n} I\left(X_{t} \leq x\right), x \in \mathbb{R}$, and a weighting function $J:[0,1] \rightarrow \mathbb{R}$. Under the mild mixing/moment conditions used for sample means in Theorem 5, the same conclusions for subsampling and convolved subsampling also hold with general Lestimators $T_{n}=\sqrt{n}\left(t_{n}-t(P)\right)$, provided that $J$ is bounded and continuous almost everywhere; see the Supplementary Material [15] for more formal details regarding L-estimators. Section 7 provides some numerical justification of the convolved procedure for the trimmed mean as an L-statistic.

6. Independent data versions. For completeness, we briefly mention a variation of convolved subsampling for independent data. Recall that Section 4.1 considered block-based convolved subsampling with general statistics computed from strongly mixing time processes $\left\{X_{t}\right\}$. Hence, results of Section 4.1 apply to independent data, as do block bootstrap results of Section 4.2 for sample means under mixing conditions. However, with independent $X_{1}, \ldots, X_{n}$, one may consider a different formulation of subsamples rather than data blocks of $b$ consecutive observations. Namely, let $b_{n} \equiv b$ denote a set size and define subsamples $Y_{b, 1}, \ldots, Y_{b, N_{n}}$ as the $N_{n} \equiv\left(\begin{array}{l}n \\ b\end{array}\right)$ unordered subsets of size $b$ from $\left\{X_{1}, \ldots, X_{n}\right\}$. The "independent data" subsampling estimator $S_{n, \mathrm{SUB}}^{\mathrm{ID}}$ is defined as $S_{n, \mathrm{SUB}}$ in (2.1) with subsample statistics $t_{n, b, i} \equiv t_{b}\left(Y_{b, i}\right), i=1, \ldots, N_{n}$, where statistics $t_{b}(\cdot)$ are symmetric in their arguments here; see Politis, Romano and Wolf [29] (Chapter 2) for a general treatment of this subsampling estimator with i.i.d. data.

The next theorem verifies that, for independent data, the general results for convolved subsampling with previous block-based subsamples (Section 4.1) also hold when the convolution is based on the independent data subsampling estimator $S_{n, \mathrm{SUB}}^{\mathrm{ID}}$ using all subsets of size $b$.

THEOREM 6. Let $\left\{X_{t}\right\}$ be a sequence of independent (possibly non-i.i.d.) random variables. Given $S_{n, \mathrm{SUB}}^{\mathrm{ID}}$, let $\left(\hat{\sigma}_{n, \mathrm{SUB}}^{\mathrm{ID}}\right)^{2}$ and $C_{n, k_{n}}^{\mathrm{ID}}$ denote the corresponding subsampling variance estimator and convolved subsampling estimator. Then Theorem 4 holds under the notational convention that $S_{n, \mathrm{SUB}} \equiv S_{n, \mathrm{SUB}}^{\mathrm{ID}}, \hat{\sigma}_{n, \mathrm{SUB}}^{2} \equiv$ 
$\left(\hat{\sigma}_{n, \mathrm{SUB}}^{\mathrm{ID}}\right)^{2}$, and $C_{n, k_{n}} \equiv C_{n, k_{n}}^{\mathrm{ID}}$ and that subsample quantities in (3.1)-(3.2) are defined as $T_{b, i}=\tau_{b}\left[t_{b}\left(Y_{b, i}\right)-t(P)\right], i=1, \ldots, N_{n} \equiv\left(\begin{array}{l}n \\ b\end{array}\right)$.

Additionally, if the variables $\left\{X_{t}\right\}$ are i.i.d., then Corollary 2 likewise holds.

We may also draw some connections between convolved subsampling and the bootstrap for sample means with independent data. Suppose independent variables $X_{1}, \ldots, X_{n}$ have common mean $\mu$ (e.g., as in Tukey's symmetric contamination model where observations may have different variances), from which we define $T_{n} \equiv \sqrt{n}\left(\bar{X}_{n}-\mu\right)$. The convolved estimator $C_{n, k_{n}}^{\mathrm{ID}}$ here has close parallels to the classic independent bootstrap of Efron [11]. Namely, $C_{n, k_{n}}^{\mathrm{ID}}$ is the resampling distribution of $T_{n}^{*} \equiv \sqrt{n_{1}}\left(\bar{X}_{n_{1}}^{*}-\bar{X}_{n}\right)$ for a sample mean $\bar{X}_{n_{1}}^{*}$ of size $n_{1}=k_{n} b$ formed by averaging $k_{n}$ independent subsamples of size $b$, with each size $b$ subsample drawn uniformly and without replacement from $\left\{X_{i}\right\}_{i=1}^{n}$; if the subsamples of size $b$ are instead drawn with replacement from $\left\{X_{i}\right\}_{i=1}^{n}$, then $T_{n}^{*}$ alternatively produces the independent bootstrap distribution, say $C_{n, k_{n} b}^{\mathrm{ID}, \text { boot }}$, with a resample size $n_{1}=k_{n} b$. Consequently, the independent data version of convolved subsampling $C_{n, k_{n}}^{\mathrm{ID}}$ does not exactly match the independent bootstrap. However, the following result for independent data shows that the subsampling estimator $S_{n, \mathrm{SUB}}^{\mathrm{ID}}$ and its convolution $C_{n, k_{n}}^{\mathrm{ID}}$ are valid in a broad non-i.i.d. context for sample means, and the differences between $C_{n, k_{n}}^{\mathrm{ID}}$ and $C_{n, k_{n} b}^{\mathrm{ID} \text {,bot }}$ are asymptotically negligible.

THEOREM 7. Let $X_{1}, X_{2}, \ldots$, denote a sequence of independent (possibly non-i.i.d.) variables, with finite variances and common mean $\mathrm{E} X_{t}=\mu \in \mathbb{R}$. Define $X_{i, \mu} \equiv X_{i}-\mu, i \geq 1$. As $n \rightarrow \infty$, suppose $b^{-1}+b / n \rightarrow 0$ and that

$$
\frac{1}{n} \sum_{i=1}^{n} \mathrm{E} X_{i, \mu}^{2} I\left[\left|X_{i, \mu}\right|>\epsilon \sqrt{b}\right] \rightarrow 0
$$

and

$$
\max _{1 \leq i_{1}<i_{2}<\cdots<i_{b} \leq n}\left|\frac{1}{b} \sum_{j=1}^{b} \mathrm{E} X_{i_{j}, \mu}^{2}-\sigma^{2}\right| \rightarrow 0
$$

for each $\epsilon>0$ and some $\sigma^{2}>0$. Then, as $n \rightarrow \infty, T_{n}=\sqrt{n}\left(\bar{X}_{n}-\mu\right) \stackrel{d}{\rightarrow} N\left(0, \sigma^{2}\right)$ along with $\sup _{x \in \mathbb{R}}\left|S_{n, \mathrm{SUB}}^{\mathrm{ID}}(x)-\Phi(x / \sigma)\right| \stackrel{p}{\rightarrow} 0$ and $\left(\hat{\sigma}_{n, \mathrm{SUB}}^{\mathrm{ID}}\right)^{2} \stackrel{p}{\rightarrow} \sigma^{2}$. Furthermore, for any positive integer sequence $k_{n}$,

$$
\sup _{x \in \mathbb{R}}\left|C_{n, k_{n}}^{\mathrm{ID}}(x)-\Phi(x / \sigma)\right| \stackrel{p}{\rightarrow} 0 \quad \text { and } \quad d_{2}\left[C_{n, k_{n}}^{\mathrm{ID}}, C_{n, k_{n} b}^{\mathrm{ID}, \mathrm{boot}}\right] \stackrel{p}{\rightarrow} 0
$$

where $d_{2}(\cdot, \cdot)$ denotes Mallow's metric between distributions $C_{n, k_{n}}^{\mathrm{ID}}$ and $C_{n, k_{n} b}^{\mathrm{ID} b \text { bot }}$. 
REMARK 4. Above $d_{2}(\cdot, \cdot)$ metricizes weak convergence [5], where, for distributions $F$ and $G$ on $\mathbb{R},\left[d_{2}(F, G)\right]^{2} \equiv \inf \left\{\mathrm{E}|X-Y|^{2}: X \sim F, Y \sim G\right\}$ with the infimum over all pairs $(X, Y)$ with marginal distributions $F$ and $G$.

A Lindeberg condition, defined by replacing $b$ with $n$ in the second moment assumptions of Theorem 7 , suffices for $T_{n}=\sqrt{n}\left(\bar{X}_{n}-\mu\right) \stackrel{d}{\rightarrow} N\left(0, \sigma^{2}\right)$. Hence, Theorem 7 validates subsampling, convolved subsampling and the bootstrap for the sample mean under a slightly stronger condition than required for the CLT with independent data. This finding also involves a weaker moment condition than a classical bootstrap result of Liu [24] for sample means of non-i.i.d. data (i.e., $\sup _{i \geq 1} \mathrm{E}\left|X_{i}\right|^{2+\delta}<\infty$ with $\delta>0$ ). Also, for general statistics with i.i.d. data, Politis, Romano and Wolf [29] (Corollary 2.3.1) use the subsampling estimator $S_{n, \text { ID }}^{\text {ID }}$ to prove the consistency of a version of the bootstrap with a resample size of $b<n$, provided that $b^{-1}+b^{2} / n \rightarrow 0$. When specialized to sample means, their intended bootstrap becomes $C_{n, k_{n} b}^{\mathrm{ID} \text {,bot }}$ with convolution $k_{n}=1$ and Theorem 7 generalizes their result under a weaker requirement $b / n \rightarrow 0$ and for non-i.i.d. data.

7. Numerical results. Particularly in the important case of the sample mean, where convolved subsampling equals the classical block bootstrap, many authors have shown, via numerical as well as theoretical evidence, that the bootstrap is generally advantageous over standard subsampling when both are valid (cf. [19], Chapter 6; [29], Chapter 10). Intuitively, the i.i.d. randomization of the bootstrap often better aligns its distribution with a normal target. Hence, when considering the sample mean, our aim here is of theoretical nature and we provide a new approach for proving bootstrap consistency.

For statistics of interest beyond the sample mean, the situation is different. In this scenario, convolved subsampling estimation gives a completely new resampling procedure, where results from Section 3 can be used to establish the method's consistency in general contexts. As described in Section 5, the method has been applied to spectral densities [21] and U-statistics [32], where some numerical findings have indicated improvements over subsampling with U-statistics. Outside of this, not much is presently known about the method's properties relative to subsampling or block bootstrap. However, for approximating normal limits, convolved subsampling might generally be anticipated to perform better than subsampling, as convolution may move estimators closer to normality (cf. Remark 5 to follow).

For comparison, we include a small simulation study involving the trimmed mean statistic (cf. Section 5.3), computed from a time series $X_{1}, \ldots, X_{n}$ as

$$
\bar{X}_{n, \tau_{1}, \tau_{2}}=\frac{1}{\left\lfloor n \tau_{2}\right\rfloor-\left\lfloor n \tau_{1}\right\rfloor} \sum_{i=\left\lfloor n \tau_{1}\right\rfloor+1}^{\left\lfloor n \tau_{2}\right\rfloor} X_{(i)},
$$

using order statistics $X_{(1)}, \ldots, X_{(n)}$ and trimming proportions $0 \leq \tau_{1}<\tau_{2} \leq 1$. When $\tau_{1}=0$ and $\tau_{2}=1$, the trimmed mean equals the sample mean and, conse- 
quently, convolved subsampling becomes equivalent to the block bootstrap. However, when observations are discarded by $\tau_{1} \neq 0$ or $\tau_{2} \neq 1$, the two resampling procedures are different. For the trimmed mean parameter, $90 \%$ confidence intervals are given by $\bar{X}_{n, \tau_{1}, \tau_{2}} \pm q_{0.9} / \sqrt{n}$, with $q_{0.9}$ denoting an appropriate quantile found by either subsampling, convolved subsampling with different levels of convolution, or the moving blocks bootstrap. For data generation, we use an AR(1)process with standard normal marginals.

Table 1 displays the coverage probabilities from intervals by the aforementioned resampling methods, based on an $\mathrm{AR}(1)$ parameter 0.3 and various sample sizes $n$;

TABLE 1

Coverage percentages of $90 \%$ intervals for the trimmed mean based on subsampling $\left(S_{n, \mathrm{SUB}}\right)$, convolved subsampling $\left(C_{n, k_{n}}\right)$ and block bootstrap $\left(C_{n,\lfloor n / b\rfloor}^{\text {boot }}\right)$ for various block sizes $b$, convolution levels $k_{n}$ and sample sizes $n$; coverages are based on 1000 simulations from an $A R(1)$-process (coefficient 0.3 ) with distributions for $C_{n, k_{n}}$ or $C_{n,\lfloor n / b\rfloor}^{\text {boot }}$ approximated from 999 resamples. Note $C_{n,\lfloor n / b\rfloor}$ and $C_{n,\lfloor n / b\rfloor}^{\text {boot }}$ match for $\tau_{1}=1, \tau_{2}=0$

\begin{tabular}{|c|c|c|c|c|c|c|c|c|c|c|}
\hline \multirow{2}{*}{$\begin{array}{l}n \\
b\end{array}$} & \multicolumn{2}{|c|}{50} & \multicolumn{2}{|c|}{100} & \multicolumn{2}{|c|}{200} & \multicolumn{2}{|c|}{500} & \multicolumn{2}{|c|}{1000} \\
\hline & 7 & 3 & 10 & 4 & 14 & 5 & 22 & 7 & 31 & 9 \\
\hline & \multicolumn{10}{|c|}{ Percentages of trimming $\tau_{1}=1-\tau_{2}=0$} \\
\hline$S_{n, \mathrm{SUB}}$ & 79.1 & 84.5 & 84.2 & 86.3 & 85.0 & 86.9 & 89.3 & 88.9 & 87.3 & 89.1 \\
\hline$C_{n, 3}$ & 80.1 & 84.8 & 85.3 & 86.5 & 84.2 & 87.2 & 89.4 & 88.5 & 87.3 & 88.8 \\
\hline$C_{n,\lfloor n /(2 b)\rfloor}$ & 80.3 & 85.0 & 86.1 & 86.7 & 85.3 & 87.5 & 89.4 & 89.1 & 87.7 & 89.3 \\
\hline$C_{n,\lfloor n / b\rfloor}$ & 82.0 & 85.5 & 86.4 & 87.4 & 85.5 & 87.8 & 89.4 & 89.2 & 87.6 & 89.9 \\
\hline \multirow[t]{2}{*}{$C_{n,\lfloor n / b\rfloor}^{\text {boot }}$} & 82.0 & 85.5 & 86.4 & 87.4 & 85.5 & 87.8 & 89.4 & 89.2 & 87.6 & 89.9 \\
\hline & \multicolumn{10}{|c|}{ Percentages of trimming $\tau_{1}=1-\tau_{2}=0.05$} \\
\hline$S_{n, \mathrm{SUB}}$ & 82.4 & 86.3 & 83.9 & 87.6 & 84.3 & 88.4 & 88.0 & 87.1 & 87.3 & 87.3 \\
\hline$C_{n, 3}$ & 82.3 & 85.9 & 84.4 & 88.4 & 83.7 & 89.1 & 88.0 & 86.7 & 87.4 & 87.8 \\
\hline$C_{n,\lfloor n /(2 b)\rfloor}$ & 82.5 & 86.2 & 84.7 & 87.9 & 84.2 & 89.2 & 88.0 & 87.7 & 87.4 & 88.0 \\
\hline$C_{n,\lfloor n / b\rfloor}$ & 84.2 & 87.4 & 85.1 & 88.7 & 84.3 & 89.8 & 88.3 & 88.1 & 87.6 & 87.7 \\
\hline \multirow[t]{2}{*}{$C_{n,\lfloor n / b\rfloor}^{\text {boot }}$} & 87.2 & 81.3 & 84.9 & 85.7 & 86.0 & 85.7 & 89.4 & 89.6 & 88.4 & 88.9 \\
\hline & \multicolumn{10}{|c|}{ Percentages of trimming $\tau_{1}=1-\tau_{2}=0.10$} \\
\hline$S_{n, \mathrm{SUB}}$ & 80.5 & 85.3 & 83.8 & 85.2 & 84.9 & 86.4 & 87.2 & 86.7 & 88.5 & 89.1 \\
\hline$C_{n, 3}$ & 80.5 & 86.4 & 83.8 & 85.4 & 84.6 & 86.9 & 87.1 & 87.1 & 88.7 & 88.9 \\
\hline$C_{n,\lfloor n /(2 b)\rfloor}$ & 80.7 & 86.5 & 84.5 & 85.6 & 84.9 & 87.7 & 86.8 & 87.0 & 88.3 & 89.2 \\
\hline$C_{n,\lfloor n / b\rfloor}$ & 82.2 & 87.3 & 85.4 & 85.9 & 86.1 & 88.0 & 88.0 & 87.9 & 88.2 & 89.4 \\
\hline \multirow[t]{2}{*}{$C_{n,\lfloor n / b\rfloor}^{\text {boot }}$} & 82.4 & 82.1 & 85.3 & 84.8 & 84.7 & 86.7 & 88.8 & 86.4 & 89.5 & 87.8 \\
\hline & \multicolumn{10}{|c|}{ Percentages of trimming $\tau_{1}=1-\tau_{2}=0.25$} \\
\hline$S_{n, \mathrm{SUB}}$ & 80.3 & 84.3 & 85.0 & 85.9 & 84.1 & 84.1 & 86.2 & 85.2 & 85.8 & 88.0 \\
\hline$C_{n, 3}$ & 80.7 & 85.1 & 85.7 & 86.2 & 84.5 & 84.2 & 86.5 & 85.0 & 86.1 & 87.0 \\
\hline$C_{n,\lfloor n /(2 b)\rfloor}$ & 81.7 & 86.0 & 85.8 & 86.6 & 85.4 & 85.1 & 86.3 & 85.3 & 86.3 & 87.7 \\
\hline$C_{n,\lfloor n / b\rfloor}$ & 82.8 & 86.3 & 86.8 & 87.8 & 85.9 & 85.9 & 87.0 & 85.9 & 86.5 & 88.9 \\
\hline$C_{n,\lfloor n / b\rfloor}^{\text {boot }}$ & 82.6 & 81.8 & 84.8 & 86.7 & 87.1 & 87.6 & 88.6 & 86.7 & 88.9 & 89.9 \\
\hline
\end{tabular}


TABLE 2

Lengths of $90 \%$ intervals for the trimmed mean corresponding to the coverage probabilities reported in Table 1

\begin{tabular}{|c|c|c|c|c|c|c|c|c|c|c|}
\hline \multirow{2}{*}{$\begin{array}{l}n \\
b\end{array}$} & \multicolumn{2}{|c|}{50} & \multicolumn{2}{|c|}{100} & \multicolumn{2}{|c|}{200} & \multicolumn{2}{|c|}{500} & \multicolumn{2}{|c|}{1000} \\
\hline & 7 & 3 & 10 & 4 & 14 & 5 & 22 & 7 & 31 & 9 \\
\hline & \multicolumn{10}{|c|}{ Percentages of trimming $\tau_{1}=1-\tau_{2}=0$} \\
\hline$S_{n, \mathrm{SUB}}$ & 0.570 & 0.606 & 0.423 & 0.435 & 0.309 & 0.315 & 0.202 & 0.202 & 0.144 & 0.144 \\
\hline$C_{n, 3}$ & 0.598 & 0.635 & 0.438 & 0.452 & 0.314 & 0.322 & 0.205 & 0.206 & 0.145 & 0.146 \\
\hline$C_{n,\lfloor n /(2 b)\rfloor}$ & 0.580 & 0.623 & 0.430 & 0.445 & 0.310 & 0.318 & 0.203 & 0.204 & 0.145 & 0.145 \\
\hline$C_{n,\lfloor n / b\rfloor}$ & 0.578 & 0.615 & 0.425 & 0.440 & 0.306 & 0.315 & 0.202 & 0.202 & 0.144 & 0.144 \\
\hline \multirow[t]{2}{*}{$C_{n,\lfloor n / b\rfloor}^{\text {boot }}$} & 0.578 & 0.615 & 0.425 & 0.440 & 0.306 & 0.315 & 0.202 & 0.202 & 0.144 & 0.144 \\
\hline & \multicolumn{10}{|c|}{ Percentages of trimming $\tau_{1}=1-\tau_{2}=0.05$} \\
\hline$S_{n, \mathrm{SUB}}$ & 0.567 & 0.605 & 0.430 & 0.436 & 0.310 & 0.315 & 0.202 & 0.202 & 0.144 & 0.145 \\
\hline$C_{n, 3}$ & 0.595 & 0.633 & 0.444 & 0.450 & 0.316 & 0.321 & 0.204 & 0.205 & 0.146 & 0.146 \\
\hline$C_{n,|n /(2 b)|}$ & 0.576 & 0.623 & 0.437 & 0.444 & 0.312 & 0.317 & 0.203 & 0.203 & 0.145 & 0.145 \\
\hline$C_{n,\lfloor n / b\rfloor}$ & 0.575 & 0.614 & 0.432 & 0.439 & 0.310 & 0.315 & 0.201 & 0.203 & 0.144 & 0.145 \\
\hline \multirow[t]{2}{*}{$C_{n,\lfloor n / b\rfloor}^{\text {boot }}$} & 0.594 & 0.580 & 0.446 & 0.425 & 0.317 & 0.309 & 0.205 & 0.200 & 0.146 & 0.144 \\
\hline & \multicolumn{10}{|c|}{ Percentages of trimming $\tau_{1}=1-\tau_{2}=0.10$} \\
\hline & 0.571 & 0.605 & 0.423 & 0.432 & 0.313 & 0.314 & 0.202 & 0.202 & 0.147 & 0.144 \\
\hline$C_{n, 3}$ & 0.597 & 0.636 & 0.437 & 0.450 & 0.320 & 0.321 & 0.205 & 0.204 & 0.148 & 0.146 \\
\hline$C_{n,\lfloor n /(2 b}$ & 0.577 & 0.624 & 0.428 & 0.443 & 0.315 & 0.318 & 0.203 & 0.203 & 0.147 & 0.145 \\
\hline$C_{n,\lfloor n / b\rfloor}$ & 0.578 & 0.615 & 0.425 & 0.437 & 0.312 & 0.314 & 0.202 & 0.202 & 0.147 & 0.144 \\
\hline \multirow[t]{2}{*}{$C_{n,\lfloor n / b\rfloor}^{\text {boot }}$} & 0.603 & 0.586 & 0.442 & 0.430 & 0.322 & 0.311 & 0.206 & 0.202 & 0.149 & 0.145 \\
\hline & \multicolumn{10}{|c|}{ Percentages of trimming $\tau_{1}=1-\tau_{2}=0.25$} \\
\hline$S_{n, \mathrm{SUB}}$ & 0.571 & 0.607 & 0.432 & 0.437 & 0.323 & 0.315 & 0.209 & 0.203 & 0.151 & 0.148 \\
\hline$C_{n, 3}$ & 0.602 & 0.647 & 0.453 & 0.460 & 0.333 & 0.329 & 0.214 & 0.210 & 0.153 & 0.152 \\
\hline$C_{n,\lfloor n /(2 b)\rfloor}$ & 0.578 & 0.627 & 0.440 & 0.448 & 0.326 & 0.322 & 0.211 & 0.206 & 0.151 & 0.150 \\
\hline$C_{n,\lfloor n / b\rfloor}$ & 0.578 & 0.614 & 0.434 & 0.439 & 0.323 & 0.316 & 0.209 & 0.203 & 0.150 & 0.148 \\
\hline$C_{n,\lfloor n / b\rfloor}^{\text {boot }}$ & 0.623 & 0.614 & 0.459 & 0.451 & 0.335 & 0.326 & 0.214 & 0.211 & 0.153 & 0.151 \\
\hline
\end{tabular}

qualitatively similar results with other AR parameters appear in the Supplementary Material [15]. Additionally, corresponding interval lengths are reported in Table 2. As those differ only marginally over the five resampling methods, we will focus on the probabilities. In general, all methods tend to yield coverage probabilities below the nominal level of $90 \%$. Moreover, convolution of subsampling improves performance over regular subsampling in almost all cases, with increasing levels of convolution tending to produce better coverage accuracy. For illustration, the subsampling distributional approximation for the trimmed mean statistic is shown in Figure 1 along with counterparts improved by convolution. In terms of comparisons between the block bootstrap and convolved subsampling, the methods again differ when $\tau_{1} \neq 0$ or $\tau_{2} \neq 1$, as indicated in Table 1 . Based on our simulation, neither method seems to be clearly advantageous. Depending on the choice of 

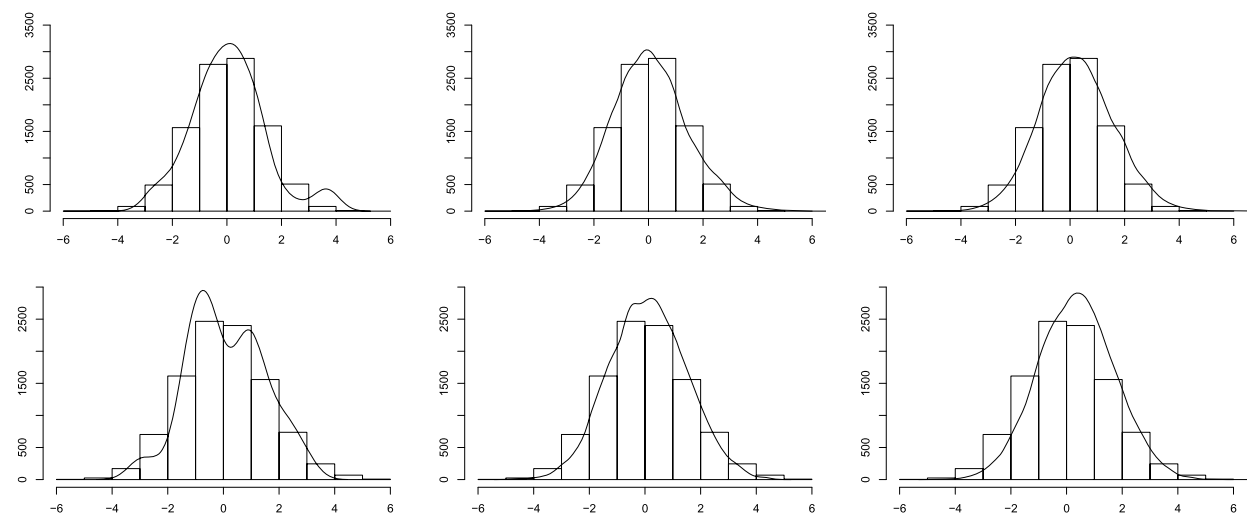

FIG. 1. Histogram of the centered/scaled trimmed mean, with $\tau_{1}=1-\tau_{2}=0.10$ (top) or $\tau_{1}=1-\tau_{2}=0.25$ (bottom), based on 10,000 simulations from an AR(1) process, and density estimates by convolved subsampling with convolution levels of $k_{n}=1$ (i.e., subsampling) (left), $k_{n}=3$ (center) and $k_{n}=14$ (right) from one data realization.

trimming parameters, block length $b$, and level of convolution $k_{n}$, either bootstrap or convolved subsampling may emerge as the better of the two.

REMARK 5. For approximating normal limits, convolved subsampling may reduce skewness in distributional estimates from basic subsampling. For illustration, considering the sample mean, the distribution of $T_{n}=\sqrt{n}\left(\bar{X}_{n}-\mu\right)$ often has approximate skewness $\gamma / \sqrt{n}$ for some constant $\gamma$. In this case, the corresponding subsampling estimator $S_{n, \text { SUB }}$ is known to have a larger approximate skewness $\gamma / \sqrt{b}$, while a more fully convolved estimator (bootstrap) $C_{n, k_{n}}$ with $k_{n} \approx n / b$ has skewness approximately $\gamma / \sqrt{n}$. A better matching skewness may improve higherorder accuracy; see [29] (Section 10.2).

8. Concluding remarks and extensions. For approximating sampling distributions with normal limits, we have developed a theory for the $k$-fold selfconvolution of subsampling estimators. Results validate the method for general statistics and dependent data structures, based on mild consistency properties of the basic subsampling estimator and its subsampling variance. The latter estimator is crucial under diverging levels of convolution, as occurs with block bootstrap. For time series, convolved subsampling matches the block bootstrap for sample means. With more general statistics, convolved subsampling often differs from the bootstrap and, instead, provides a hybrid-type of resampling that has received recent consideration (cf. Section 5) and may improve upon standard subsampling for normal targets. Further study is required of higher-order accuracy. However, as convolved subsampling can often be verified under mild process assumptions, this offers an alternative approach for establishing the block bootstrap under weaker conditions than previously considered for sample means in particular. 
We make two final points about applying convolved subsampling to validate the block bootstrap with more general statistics, which is difficult to characterize. First, from the standard linearization technique based on functional representations and differentials, a target statistic may often be decomposed into main/linear and remainder parts (cf. [17]; Chapter 4.3, [19]). Commonly, the linear term is a sample mean (e.g., of influence functions), while the remainder is negligible. Convolved subsampling can be directly used to establish the block bootstrap for this main term under weak conditions. Our framework, though, does not immediately address the remainder.

However, second, such remainders are often smaller-order than a norm of the centered empirical distribution, in both the original data and bootstrap worlds, where the bootstrap version is, say, $\sqrt{n}\left\|\hat{G}_{n}^{*}-\mathrm{E}_{*} \hat{G}_{n}^{*}\right\|$. By virtue of the empirical distribution as an average, the bootstrap estimator $\sqrt{n}\left(\hat{G}_{n}^{*}(x)-\mathrm{E}_{*} \hat{G}_{n}^{*}(x)\right)$, $x \in \mathbb{R}$, must consequently match convolved subsampling. This connection could potentially foster bootstrap studies with empirical processes and dependent data, particularly as subsampling estimators are consistent for empirical processes under weak assumptions ([29], Chapter 7.4). Further investigation is required, though, because the technical obstacle to weak convergence differs substantially from the usual sample mean case: namely, tightness of the bootstrap/convolved subsampling process $\sqrt{n}\left(\hat{G}_{n}^{*}(\cdot)-\mathrm{E}_{*} \hat{G}_{n}^{*}(\cdot)\right)$ must be determined on a suitable metric space (cf. [19], Chapter 4.4.1). Still, convolved subsampling may provide a general tool for advancing future developments with resampling dependent data.

Acknowledgments. The authors are grateful to the editors and two referees for their time and thoughtful comments, which improved the manuscript.

\section{SUPPLEMENTARY MATERIAL}

Supplement to "Convolved subsampling estimation with applications to block bootstrap” (DOI: 10.1214/18-AOS1695SUPP; .pdf). This supplement provides proofs for the distributional results about convolved subsampling and further numerical/theoretical support for the simulation study.

\section{REFERENCES}

[1] AthreyA, K. B. and LAhIRI, S. N. (2006). Measure Theory and Probability Theory. Springer Texts in Statistics. Springer, New York. MR2247694

[2] BAI, S. and TAQQU, M. S. (2017). On the validity of resampling methods under long memory. Ann. Statist. 45 2365-2399. MR3737895

[3] Beran, J. (1994). Statistics for Long-Memory Processes. Monographs on Statistics and Applied Probability 61. Chapman \& Hall, New York. MR1304490

[4] BetKen, A. and Wendler, M. (2018). Subsampling for general statistics under long range dependence. Statist. Sinica. 28 1199-1224. MR3821001

[5] Bickel, P. J. and Freedman, D. A. (1981). Some asymptotic theory for the bootstrap. Ann. Statist. 9 1196-1217. MR0630103 
[6] Cambanis, S., Houdré, C., Hurd, H. and LeŚkow, J. (1994). Laws of large numbers for periodically and almost periodically correlated processes. Stochastic Process. Appl. $\mathbf{5 3}$ 37-54. MR1290706

[7] Corduneanu, C. (1989). Almost Periodic Functions. Chelsea, New York.

[8] DaVydov, Y. A. (1970). The invariance principle for stationary processes. Theory Probab. Appl. 15 487-498.

[9] Dehling, H. and Wendler, M. (2010). Central limit theorem and the bootstrap for $U$-statistics of strongly mixing data. J. Multivariate Anal. 101 126-137. MR2557623

[10] Dobrushin, R. L. and MAJOR, P. (1979). Non-central limit theorems for nonlinear functionals of Gaussian fields. Z. Wahrsch. Verw. Gebiete 50 27-52. MR0550122

[11] Efron, B. (1979). Bootstrap methods: Another look at the jackknife. Ann. Statist. 7 1-26. MR0515681

[12] Fitzenberger, B. (1998). The moving blocks bootstrap and robust inference for linear least squares and quantile regressions. J. Econometrics 82 235-287. MR1613422

[13] Hosking, J. R. M. (1981). Fractional differencing. Biometrika 68 165-176. MR0614953

[14] HuRD, H. L. (1991). Correlation theory of almost periodically correlated processes. J. Multivariate Anal. 37 24-45. MR1097303

[15] Tewes, J., Politis, D. N. and Nordman, D. J. (2019). Supplement to "Convolved subsampling estimation with applications to block bootstrap." DOI:10.1214/18-AOS1695SUPP.

[16] Kim, Y. M. and Nordman, D. J. (2011). Properties of a block bootstrap under long-range dependence. Sankhya A 73 79-109. MR2887088

[17] KÜNSCH, H. R. (1989). The jackknife and the bootstrap for general stationary observations. Ann. Statist. 17 1217-1241. MR1015147

[18] LAHIRI, S. N. (1993). On the moving block bootstrap under long range dependence. Statist. Probab. Lett. 18 405-413. MR1247453

[19] LahiRI, S. N. (2003). Resampling Methods for Dependent Data. Springer Series in Statistics. Springer, New York. MR2001447

[20] LAHIRI, S. N. (2003). Central limit theorems for weighted sums of a spatial process under a class of stochastic and fixed designs. Sankhya 65 356-388. MR2028905

[21] LenarT, Ł. (2011). Asymptotic distributions and subsampling in spectral analysis for almost periodically correlated time series. Bernoulli 17 290-319. MR2797993

[22] LENART, Ł. (2016). Generalized resampling scheme with application to spectral density matrix in almost periodically correlated class of times series. J. Time Series Anal. 37 369-404. MR3512963

[23] Leucht, A. (2012). Degenerate $U$ - and $V$-statistics under weak dependence: Asymptotic theory and bootstrap consistency. Bernoulli 18 552-585. MR2922461

[24] LiU, R. Y. (1988). Bootstrap procedures under some non-i.i.d. models. Ann. Statist. 16 16961708. MR0964947

[25] LIU, R. Y. and SiNGH, K. (1992). Moving blocks jackknife and bootstrap capture weak dependence. In Exploring the Limits of Bootstrap (East Lansing, MI, 1990). Wiley Ser. Probab. Math. Statist. Probab. Math. Statist. 225-248. Wiley, New York. MR1197787

[26] Mandelbrot, B. B. and Van Ness, J. W. (1968). Fractional Brownian motions, fractional noises and applications. SIAM Rev. 10 422-437. MR0242239

[27] Nordman, D. J. and LahiRI, S. N. (2005). Validity of the sampling window method for long-range dependent linear processes. Econometric Theory 21 1087-1111. MR2200986

[28] Politis, D. N. and Romano, J. P. (1994). Large sample confidence regions based on subsamples under minimal assumptions. Ann. Statist. 22 2031-2050. MR1329181

[29] Politis, D. N., Romano, J. P. and Wolf, M. (1999). Subsampling. Springer Series in Statistics. Springer, New York. MR1707286

[30] RADULOVIĆ, D. (1996). The bootstrap of the mean for strong mixing sequences under minimal conditions. Statist. Probab. Lett. 28 65-72. MR1394420 
[31] RAdulović, D. (2012). Necessary and sufficient conditions for the moving blocks bootstrap central limit theorem of the mean. J. Nonparametr. Stat. 24 343-357. MR2921140

[32] Sharipov, O. Sh., Tewes, J. and Wendler, M. (2016). Bootstrap for $U$-statistics: A new approach. J. Nonparametr. Stat. 28 576-594. MR3514512

[33] Sharipov, O. Sh. and Wendler, M. (2012). Bootstrap for the sample mean and for $U$-statistics of mixing and near-epoch dependent processes. J. Nonparametr. Stat. $24317-$ 342. MR2921139

[34] SYNOwIECKI, R. (2007). Consistency and application of moving block bootstrap for nonstationary time series with periodic and almost periodic structure. Bernoulli 13 11511178. MR2364230

[35] TAQQU, M. S. (1974/75). Weak convergence to fractional Brownian motion and to the Rosenblatt process. Z. Wahrsch. Verw. Gebiete 31 287-302. MR0400329

[36] Zhang, T., Ho, H.-C., Wendler, M. and Wu, W. B. (2013). Block sampling under strong dependence. Stochastic Process. Appl. 123 2323-2339. MR3038507

\section{J. TEWES}

DEPARTMENT OF MATHEMATiCs

RUHR-UNIVERSITÄT BOCHUM

44780 BOCHUM

GERMANY

E-MAIL: johannes.tewes@ruhr-uni-bochum.de

\section{N. POLITIS}

DEPARTMENT OF MATHEMATICS

University of CALIFornia, SAN Diego

LA Jolla, CALIFORNIA 92093-0112

USA

E-MAIL: dpolitis@ucsd.edu

D. J. NORDMAN

DEPARTMENT OF STATISTICS

IOWA STATE UNIVERSITY

AMES, IOWA 50011

USA

E-MAIL: dnordman@iastate.edu 